\title{
On the Generalized Lognormal Distribution
}

\author{
Thomas L. Toulias and Christos P. Kitsos \\ Technological Educational Institute of Athens, Department of Mathematics, Ag. Spyridonos \& Palikaridi Street, \\ 12210 Egaleo, Athens, Greece \\ Correspondence should be addressed to Thomas L. Toulias; t.toulias@teiath.gr
}

Received 11 April 2013; Revised 4 June 2013; Accepted 5 June 2013

Academic Editor: Mohammad Fraiwan Al-Saleh

Copyright (C) 2013 T. L. Toulias and C. P. Kitsos. This is an open access article distributed under the Creative Commons Attribution License, which permits unrestricted use, distribution, and reproduction in any medium, provided the original work is properly cited.

\begin{abstract}
This paper introduces, investigates, and discusses the $\gamma$-order generalized lognormal distribution ( $\gamma$-GLD). Under certain values of the extra shape parameter $\gamma$, the usual lognormal, log-Laplace, and log-uniform distribution, are obtained, as well as the degenerate Dirac distribution. The shape of all the members of the $\gamma$-GLD family is extensively discussed. The cumulative distribution function is evaluated through the generalized error function, while series expansion forms are derived. Moreover, the moments for the $\gamma$ GLD are also studied.
\end{abstract}

\section{Introduction}

Lognormal distribution has been widely applied in many different aspects of life sciences, including biology, ecology, geology, and meteorology as well as in economics, finance, and risk analysis, see [1]. Also, it plays an important role in Astrophysics and Cosmology; see [2-4] among others, while for Lognormal expansions see [5].

In principle, the lognormal distribution is defined as the distribution of a random variable whose logarithm is normally distributed, and usually it is formulated with two parameters. Furthermore, log-uniform and log-laplace distributions can be similarly defined with applications in finance; see $[6,7]$. Specifically, the power-tail phenomenon of the Log-Laplace distributions [8] attracts attention quite often in environmental sciences, physics, economics, and finance as well as in longitudinal studies [9]. Recently, Log-Laplace distributions have been proposed for modeling growth rates as stock prices [10] and currency exchange rates [7].

In this paper a generalized form of Lognormal distribution is introduced, involving a third shape parameter. With this generalization, a family of distributions is emerged, which combines theoretically all the properties of Lognormal, Log-Uniform, and Log-Laplace distributions, depending on the value of this third parameter.
The generalized $\gamma$-order Lognormal distribution $(\gamma$-GLD) is the distribution of a random vector whose logarithm follows the $\gamma$-order normal distribution, an exponential power generalization of the usual normal distribution, introduced by $[11,12]$. This family of $p$-dimensional generalized normal distributions, denoted by $\mathscr{N}_{\gamma}^{p}(\mu, \Sigma)$, is equipped with an extra shape parameter $\gamma$ and constructed to play the role of normal distribution for the generalized Fisher's entropy type of information; see also $[13,14]$.

The density function $f_{X}$ of a $p$-variate, $\gamma$-order, normally distributed random variable $Y \sim \mathcal{N}_{\gamma}^{p}(\mu, \Sigma)$, with location vector $\mu \in \mathbb{R}^{p}$, positive definite scale matrix $\Sigma \in \mathbb{R}^{p \times p}$, and shape parameter $\gamma \in \mathbb{R} \backslash[0,1]$, is given by [11].

$$
\begin{aligned}
& f_{Y}(y)=f_{Y}(y ; \mu, \Sigma, \gamma) \\
&=C_{\gamma}^{p}|\operatorname{det} \Sigma|^{-1 / 2} \exp \left\{-\frac{\gamma-1}{\gamma} Q_{\theta}(y)^{\gamma / 2(\gamma-1)}\right\}, \\
& y \in \mathbb{R}^{p},
\end{aligned}
$$

where $Q_{\theta}$ is the quadratic form $Q_{\theta}(y)=(y-\mu)^{\mathrm{T}} \Sigma^{-1}(y-\mu)$, $\theta=(\mu, \Sigma)$, while $C_{\gamma}^{p}$ being the normalizing factor

$$
C_{\gamma}^{p}=\pi^{-p / 2} \frac{\Gamma(p / 2+1)}{\Gamma(p((\gamma-1) / \gamma))}\left(\frac{\gamma-1}{\gamma}\right)^{p((\gamma-1) / \gamma)-1} .
$$


From (1), notice that the second-ordered normal is the known multivariate normal distribution; that is, $\mathcal{N}_{2}^{p}(\mu, \Sigma)=$ $\mathcal{N}^{p}(\mu, \Sigma)$; see also $[13,15]$.

In Section 2, a generalized form of the Lognormal distribution is introduced, which is derived from the univariate family of $\mathcal{N}_{\gamma}\left(\mu, \sigma^{2}\right)=\mathcal{N}_{\gamma}^{1}\left(\mu, \sigma^{2}\right)$ distributions, denoted by $\mathscr{L} \mathcal{N}_{\gamma}(\mu, \sigma)$, and includes the Log-Laplace distribution as well as the Log-Uniform distribution. The shape of the $\mathscr{L} \mathcal{N}_{\gamma}(\mu, \sigma)$ members is extensively discussed while it is connected to the tailing behavior of $\mathscr{L} \mathscr{N}_{\gamma}$ through the study of the c.d.f. In Section 3, an investigation of the moments of the generalized Lognormal distribution, as well as the special cases of LogUniform and Log-Laplace distributions, is presented.

The generalized error function, that is briefly provided here, plays an important role in the development of $\mathscr{L} \mathscr{N}_{\gamma}(\mu, \sigma)$; see Section 2. The generalized error function denoted by $\operatorname{Erf}_{a}$ and the generalized complementary error function $\operatorname{Erfc}_{a}=1-\operatorname{Erf}_{a}, a \geq 0$ [16], are defined, respectively, as

$$
\operatorname{Erf}_{a}(x):=\frac{\Gamma(a+1)}{\sqrt{\pi}} \int_{0}^{x} e^{-t^{a}} d t, \quad x \in \mathbb{R}
$$

The generalized error function can be expressed (changing to $t^{a}$ variable), through the lower incomplete gamma function $\gamma(a, x)$ or the upper (complementary) incomplete gamma function $\Gamma(a, x)=\Gamma(a)-\gamma(a, x)$, in the form

$$
\begin{array}{r}
\operatorname{Erf}_{a}(x)=\frac{\Gamma(a)}{\sqrt{\pi}} \gamma\left(\frac{1}{a}, x^{a}\right)=\frac{\Gamma(a)}{\sqrt{\pi}}\left[\Gamma\left(\frac{1}{a}\right)-\Gamma\left(\frac{1}{a}, x^{a}\right)\right] \\
x \in \mathbb{R} ;
\end{array}
$$

see [16]. Moreover, adopting the series expansion form of the lower incomplete gamma function,

$$
\gamma(a, x):=\int_{0}^{x} t^{a-1} e^{-t} d t=\sum_{k=0}^{\infty} \frac{(-1)^{k}}{k !(a+k)} x^{a+k}, \quad x, a \in \mathbb{R}_{+},
$$

a series expansion form of the generalized error function is extracted:

$$
\operatorname{Erf}_{a}(x)=\frac{\Gamma(a+1)}{\sqrt{\pi}} \sum_{k=0}^{\infty} \frac{(-1)^{k}}{k !(k a+1)} x^{k a+1}, \quad x, a \in \mathbb{R}_{+} .
$$

Notice that, $\operatorname{Erf}_{2}$ is the known error function erf, that is, $\operatorname{Erf}_{2}(x)=\operatorname{erf}(x)$, while $\operatorname{Erf}_{0}$ is the function of a straight line through the origin with slope $(e \sqrt{\pi})^{-1}$. Applying $a=2$, the known incomplete gamma function identities such as $\gamma(1 / 2, x)=\sqrt{\pi} \operatorname{erf} \sqrt{x}$ and $\Gamma(1 / 2, x)=\sqrt{\pi}(1-\operatorname{erf} \sqrt{x})=$ $\sqrt{\pi} \operatorname{erfc} \sqrt{x}, x \geq 0$ are obtained. Moreover, $\operatorname{Erf}_{a} 0=0$ for all $a \in \mathbb{R}_{+}$and

$$
\lim _{x \rightarrow \pm \infty} \operatorname{Erf}_{a} x= \pm \frac{1}{\sqrt{\pi}} \Gamma(a) \Gamma\left(\frac{1}{a}\right), \quad a \in \mathbb{R}_{+},
$$

as $\gamma(a, x) \rightarrow \Gamma(a)$ when $x \rightarrow+\infty$.

\section{The $\gamma$-Order Lognormal Distribution}

The generalized univariate Lognormal distribution is defined, through the univariate generalized $\gamma$-order normal distribution, as follows.

Definition 1. When the logarithm of a random variable $X$ follows the univariate $\gamma$-order normal distribution, that is, $\log X \sim \mathcal{N}_{\gamma}\left(\mu, \sigma^{2}\right)$, then $X$ is said to follow the generalized Lognormal distribution, denoted by $\mathscr{L} \mathcal{N}_{\gamma}(\mu, \sigma)$; that is, $X \sim$ $\mathscr{L} \mathcal{N}_{\gamma}(\mu, \sigma)$.

The $\mathscr{L} \mathscr{N}_{\gamma}(\mu, \sigma)$ is referred to as the (generalized) $\gamma$-order Lognormal distribution ( $\gamma$-GLD). Like the usual Lognormal distribution, the parameter $\mu \in \mathbb{R}$ is considered to be log-scaled, while the non $\log$-scaled $\mu$ (i.e. $e^{\mu}$ when $\mu$ is assumed log-scaled) is referred to as the location parameter of $\mathscr{L} \mathscr{N}_{\gamma}(\mu, \sigma)$. Hence, if $X \sim \mathscr{L} \mathscr{N}_{\gamma}(\mu, \sigma)$, then $\log X$ is a $\gamma$-order normally distributed variable; that is, $\log X \sim \mathcal{N}_{\gamma}\left(\mu, \sigma^{2}\right)$. Therefore, the location parameter $\mu \in \mathbb{R}$ of $X$ is in fact the mean of $X$ 's natural logarithm, that is, $\mathrm{E}[\log X]=\mu$, while

$$
\begin{gathered}
\operatorname{Var}[\log X]=\left(\frac{\gamma}{\gamma-1}\right)^{2((\gamma-1) / \gamma)} \frac{\Gamma(3((\gamma-1) / \gamma))}{\Gamma(((\gamma-1) / \gamma))} \sigma^{2}, \\
\text { Kurt }[\log X]=\frac{\Gamma(\gamma-1 / \gamma) \Gamma(5(\gamma-1 / \gamma))}{\Gamma^{2}(3((\gamma-1) / \gamma))} ;
\end{gathered}
$$

see [15] for details on $\mathcal{N}_{\gamma}$.

Let $Y:=\log X \sim \mathcal{N}_{\gamma}\left(\mu, \sigma^{2}\right)$ with density function as in (1) and $X=g(Y)=e^{Y}$. Then, the density function $f_{X}$ of $X \sim \mathscr{L} \mathscr{N}_{\gamma}(\mu, \sigma)$ can be written, through (1), as

$$
\begin{aligned}
f_{X}(x) & =f_{X}(x ; \mu, \sigma, \gamma)=f_{Y}\left(g^{-1}(x)\right)\left|\frac{d}{d x} g^{-1}(x)\right| \\
& =f_{Y}(\log x) \frac{1}{x} \\
& =\frac{\exp \left\{-((\gamma-1) / \gamma)|(\log x-\mu) / \sigma|^{\gamma /(\gamma-1)}\right\}}{2 \sigma((\gamma-1) / \gamma)^{1 / \gamma} \Gamma((\gamma-1) / \gamma) x} .
\end{aligned}
$$

The probability density function $f_{X}$, as in (10), is defined in $\mathbb{R}_{+}^{*}=\mathbb{R}_{+} \backslash 0$; that is, $\mathscr{L} \mathcal{N}_{\gamma}(\mu, \sigma)$ has zero threshold. Therefore, the following definition extends Definition 1.

Definition 2. When the logarithm of a random variable $X+$ $\vartheta$ follows the univariate $\gamma$-order normal distribution, that is, $\log (X+\vartheta) \sim \mathcal{N}_{\gamma}\left(\mu, \sigma^{2}\right)$, then $X$ is said to follow the generalized Lognormal distribution with threshold $\vartheta \in \mathbb{R}$; that is, $X \sim \mathscr{L} \mathscr{N}_{\gamma}(\mu, \sigma ; \vartheta)$

It is clear that when $X \sim \mathscr{L} \mathscr{N}_{\gamma}(\mu, \sigma ; \vartheta),, \log (X-\vartheta)$ is a $\gamma$-order normally distributed variable, that is, $\log (X-\vartheta) \sim$ $\mathcal{N}_{\gamma}\left(\mu, \sigma^{2}\right)$, and thus, $\mu$ is the mean of $(X-\vartheta)$ 's natural logarithm while $\operatorname{Var}[\log X]$ is the same as in (8).

Let $Y=\log (X+\vartheta) \sim \mathcal{N}_{\gamma}\left(\mu, \sigma^{2}\right)$. The density function of $X=e^{Y}-\vartheta \sim \mathscr{L} \mathscr{N}_{\gamma}(\mu, \sigma ; \vartheta)$ is given by $f_{X}(x)=f_{X}(x-\vartheta)$, $x>0$. 
Let $z=(\log (x-9)-\mu) / \sigma$. Then, the limiting threshold density value of $f_{X}(x)$ with $x \rightarrow \vartheta^{+}$implies that

$$
\begin{aligned}
& \lim _{x \rightarrow \vartheta^{+}} f_{X}(x) \\
& \quad=\sigma^{-1} C_{\gamma_{z}}^{1} \lim _{z \rightarrow \infty} \exp \left\{-z\left(\sigma+\frac{\mu}{z}-\frac{\gamma-1}{\gamma}|z|^{1 /(\gamma-1)}\right)\right\} \\
& \quad=\sigma^{-1} C_{\gamma}^{1} e^{(\operatorname{sgn} \gamma)(-\infty)},
\end{aligned}
$$

and therefore

$$
\lim _{x \rightarrow 9^{+}} f_{X}(x)= \begin{cases}0, & \gamma \in(1,+\infty), \\ +\infty, & \gamma \in(-\infty, 0)\end{cases}
$$

that is, the $f_{X}$ 's defining domain, for the positive-ordered Lognormal random variable $X$, can be extended to include threshold point $\vartheta$ by letting $f_{X}(\vartheta)=0$.

The generalized Lognormal family of distributions $\mathscr{L} \mathscr{N}_{\gamma}$ is a wide range family bridging the Log-Uniform $\mathscr{L} \mathscr{U}$, Lognormal $\mathscr{L} \mathscr{N}$, and Log-Laplace $\mathscr{L} \mathscr{L}$ distributions, as well as the degenerate Dirac $\mathscr{D}$ distributions. We have the following.

Theorem 3. The generalized Lognormal distribution $\mathscr{L} \mathcal{N}_{\gamma}(\mu$, $\sigma)$, for order values of $\gamma=0,1,2, \pm \infty$, is reduced to

$$
\mathscr{L} \mathcal{N}_{\gamma}(\mu, \sigma)= \begin{cases}\mathscr{D}\left(e^{\mu}\right), & \gamma=0, \\ \mathscr{L} \mathscr{U}\left(e^{\mu-\sigma}, e^{\mu+\sigma}\right), & \gamma=1, \\ \mathscr{L} \mathscr{N}(\mu, \sigma), & \gamma=2, \\ \mathscr{L} \mathscr{L}\left(e^{\mu}, \frac{1}{\sigma}, \frac{1}{\sigma}\right), & \gamma= \pm \infty\end{cases}
$$

Proof. From definition (1) of $\mathcal{N}_{\gamma}$ the order $\gamma$ value is a real number outside the closed interval $[0,1]$. Let $X_{\gamma} \sim$ $\mathscr{L} \mathscr{N}_{\gamma}(\mu, \sigma)$ with density function $f_{X_{\gamma}}$ as in (10). We consider the following cases.

(i) The limiting case $\gamma=1$ : let $x \in \mathbb{R}_{+}^{*}$ such that $|\log x-\mu| \leq 1$. Using the gamma function additive identity $\Gamma(z+1)=z \Gamma(z), z \in \mathbb{R}_{+}$, in (10), we have $\mathscr{L} \mathcal{N}_{1}(\mu, \sigma)=\lim _{\gamma \rightarrow 1^{+}} \mathscr{L} \mathcal{N}_{\gamma}(\mu, \sigma)$ with

$$
\begin{aligned}
f_{X_{1}}(x) & :=\lim _{\gamma \rightarrow 1^{+}} f_{X_{\gamma}}(x) \\
& = \begin{cases}\frac{1}{2 \sigma x}, & x \in\left[e^{\mu-\sigma}, e^{\mu+\sigma}\right], \\
0, & x \in\left(-\infty, e^{\mu-\sigma}\right) \cup\left(e^{\mu+\sigma},+\infty\right),\end{cases}
\end{aligned}
$$

which is the density function of the Log-Uniform distribution $\mathscr{L} \mathscr{U}(a, b), 0<a<b$, with $a=e^{\mu-\sigma}$ and $b=$ $e^{\mu+\sigma}$; that is, $\mu=(1 / 2) \log (a b)$ and $\sigma=(1 / 2) \log (b / a)$. Therefore, first-ordered Lognormal distribution is in fact the Log-Uniform distribution, with vanishing threshold density, $f_{X_{1}}(0)=\lim _{x \rightarrow 0^{+}} f_{X_{1}}(x)=0$. For the purposes of statistical application the LogUniform moments are not the same as the model parameters; that is, although $\mu_{X}=\mu, \sigma_{X}=\sigma / \sqrt{3}$. (ii) The "normal" case $\gamma=2$ : it is clear that $\mathscr{L} \mathcal{N}_{2}(\mu, \sigma)=$ $\mathscr{L} \mathcal{N}(\mu, \sigma)$, as $f_{X_{2}}$ coincides with the Lognormal density function, and therefore the second-ordered Lognormal distribution is in fact the usual Lognormal distribution.

(iii) The limiting case $\gamma= \pm \infty$ : we have $\mathscr{L} \mathcal{N}_{ \pm \infty}(\mu, \sigma):=$ $\lim _{\gamma \rightarrow \pm \infty} \mathscr{L} \mathcal{N}_{\gamma}(\mu, \sigma)$ with

$$
\begin{aligned}
f_{X_{ \pm \infty}}(x) & :=\lim _{\gamma \rightarrow \pm \infty} f_{X_{\gamma}}(x)=\frac{1}{2 \sigma x} \exp \{-\mid \\
& = \begin{cases}\frac{e^{-\mu / \sigma}}{2 \sigma} x^{(1-\sigma) / \sigma}, & x \in\left(0, e^{\mu}\right], \\
\frac{e^{\mu / \sigma}}{2 \sigma} x^{-(\sigma+1) / \sigma}, & x>e^{\mu},\end{cases}
\end{aligned}
$$

which coincides with the density function of the known Log-Laplace distribution (symmetric logexponential distribution) $\mathscr{L} \mathscr{L}\left(\mu^{\prime}, \alpha, \beta\right)$ with $\mu^{\prime}=e^{\mu}$ and $\alpha=\beta=1 / \sigma$; see [8]. Therefore, the infiniteordered log-normal distribution is in fact the LogLaplace distribution, with threshold density

$$
f_{X_{ \pm \infty}}(0)=\lim _{x \rightarrow 0^{+}} f_{X_{ \pm \infty}}(x)= \begin{cases}0, & \sigma<1, \\ 1, & \sigma=1, \\ +\infty, & \sigma>1 .\end{cases}
$$

For the purposes of statistical application, the LogLaplace moments are not the same as the model parameters; that is, although $\mu_{X}=\mu, \sigma_{X}=\sqrt{2} \sigma$.

(iv) The limiting case $\gamma=0$ : we have

$$
\lim _{\gamma \rightarrow 0^{-}} f_{X_{\gamma}}(x)=\lim _{k:=[(\gamma-1) / \gamma] \rightarrow \infty} f_{X_{\gamma}}(x), \quad x \in \mathbb{R}_{+}^{*},
$$

where $[a]$ is the integer value of $a \in \mathbb{R}$. For the value $x=e^{\mu}$, the p.d.f. as in (10) implies

$$
\lim _{\gamma \rightarrow 0^{-}} f_{X_{\gamma}}\left(e^{\mu}\right)=\frac{1}{2 \sigma e^{\mu}}\left(\lim _{k \rightarrow \infty} \frac{k^{k}}{k !}\right) \cdot e^{0}=+\infty,
$$

through Stirling's asymptotic formula $k ! \approx \sqrt{2 \pi k}(k)$ $e)^{k}$ as $k \rightarrow \infty$. Assuming now $x \neq e^{\mu},(10)$, through (18), implies

$$
f_{X_{0}}(x):=\lim _{\gamma \rightarrow 0^{-}} f_{X_{\gamma}}(x)=\frac{|\log x-\mu|}{2 \sqrt{2 \pi} \sigma^{2} x} \cdot 0 \cdot \frac{1}{e}=0 ;
$$

that is, $\mathscr{L} \mathscr{N}_{0}(\mu, \sigma):=\lim _{\gamma \rightarrow 0^{-}} \mathscr{L} \mathcal{N}_{\gamma}(\mu, \sigma)=\mathscr{D}\left(e^{\mu}\right)$ as $f_{X_{0}}$ coincides with the Dirac density function, with the (non-log-scaled) location parameter $e^{\mu}$ of $\mathscr{L} \mathcal{N}_{0}(\mu, \sigma)$ being the singular (infinity) point. Therefore, the zero-ordered Lognormal distribution $\mathscr{L} \mathcal{N}_{0}$ is in fact the degenerate Dirac distribution with pole at the location parameter of $\mathscr{L} \mathcal{N}_{\gamma \rightarrow 0^{-}}$(with vanishing threshold density $f_{X_{0}}(0)=\lim _{x \rightarrow 0^{+}} f_{X_{0}}(x)=0$ ).

From the above limiting cases (i), (iii), and (iv), the defining domain $\mathbb{R} \backslash[0,1]$ of the order values $\gamma$, used in (1), is safely 
extended to include the values $\gamma=0,1, \pm \infty$; that is, $\gamma$ can now be defined outside the open interval $(0,1)$. Eventually, the family of the $\gamma$-order normals can include the LogUniform, Lognormal, Log-Laplace, and the degenerate Dirac distributions as (13) holds.

From Theorem 3, (12), and (15), the domain of the density functions $f_{X_{\gamma}}(x), x>0$, can also be extended to include the threshold point $x=0$ by setting $f_{X_{\gamma}}(0):=0$ for all nonnegative-ordered Lognormals, that is, for all $\gamma \in 0 \cup[1,+\infty)$, while for the Log-Laplace case of $\gamma=+\infty$ with $\sigma=1$ by setting $f_{X_{+\infty}}(0):=(1 / 2) e^{-\mu}$.

From the fact that $\mathcal{N}_{0}(\mu, \cdot)=\mathscr{D}(\mu)$, see [15], one can say that the degenerate $\log$-Dirac distribution, say $\mathscr{L} \mathscr{D}(\mu)$, equals $\mathscr{L} \mathscr{N}_{0}(\mu, \cdot)$, and hence, through Theorem 3, we can write $\mathscr{L} \mathscr{D}(\mu)=\mathscr{D}\left(e^{\mu}\right)$.

Proposition 4. The mode of the positive-ordered Lognormal random variable $X_{\gamma} \sim \mathscr{L} \mathscr{N}_{\gamma}(\mu, \sigma), X<e^{\mu} \gamma \in(1,+\infty)$, is given by

$$
\text { Mode } X_{\gamma}=e^{\mu-\sigma^{\gamma}},
$$

with corresponding maximum density value,

$$
\begin{aligned}
\max f_{X_{\gamma}} & =f_{X_{\gamma}}\left(\text { Mode } X_{\gamma}\right) \\
& =\frac{\exp \left\{\left(\sigma^{\gamma}\right) / \gamma-\mu\right\}}{2 \sigma((\gamma-1) / \gamma)^{1 / \gamma} \Gamma((\gamma-1) / \gamma)} .
\end{aligned}
$$

Proof. Recall the density function of $X_{\gamma} \sim \mathscr{L} \mathcal{N}_{\gamma}(\mu, \sigma)$ as in (10), and let $m=$ Mode $X_{\gamma}>0$. Then it holds that $(d / d x)$ $f_{X_{\gamma}}(m ; \mu, \sigma, \gamma)=0$; that is,

$$
\left(\frac{1}{\sigma}|\log m-\mu|\right)^{1 /(\gamma-1)} \frac{d}{d x}|\log x-\mu|_{x=m}=-\frac{\sigma}{m} .
$$

From $(d / d x)|x|=\operatorname{sgn} x, x \in \mathbb{R}$, we have

$$
m=e^{\mu-\sigma^{\gamma}}
$$

provided that $x<e^{\mu}$. Otherwise, (23) holds trivially, as (22) implies $\sigma=0$; that is, $m=e^{\mu}$. Moreover, $(d / d x) f_{X_{\gamma}}(x)>0$ when

$$
1+\operatorname{sgn}(\log x-\mu) \sigma^{\gamma /(1-\gamma)}|\log x-\mu|^{1 /(\gamma-1)}>0, \quad x>0,
$$

and thus, $f_{X_{\gamma}}$ is a strictly ascending density function on $\left(0, e^{\mu-\sigma^{\gamma}}\right)$ when $\gamma>1$ and also on $\left(e^{\mu-\sigma^{\gamma}}, e^{\mu}\right)$ when $\gamma<0$. Similarly, with $(d / d x) f_{X_{\gamma}}(x)<0, f_{X}$ is a strictly descending density function on $\left(e^{\mu-\sigma^{\gamma}},+\infty\right)$ when $\gamma>1$ and also on $\left(0, e^{\mu-\sigma^{\gamma}}\right) \cup\left(e^{\mu},+\infty\right)$ when $\gamma<0$. Specifically, for $\gamma<0$, the point $e^{\mu}$ is a nonsmooth point of $f_{X}$, as

$$
\begin{aligned}
\lim _{x \rightarrow e^{\mu}} \frac{d}{d x} f_{X_{\gamma}}(x)=-\frac{C_{\gamma}^{1}}{\left(\sigma e^{\mu}\right)^{2}}[\sigma & +\lim _{x \rightarrow e^{\mu}} \operatorname{sgn}(\log x-\mu) \\
& \left.\times\left|\frac{\sigma}{\log x-\mu}\right|^{1 /(1-\gamma)}\right]=+\infty .
\end{aligned}
$$

Therefore, the positive-ordered Lognormals are formed by a unimodal density function with mode as in (20) and corresponding maximum density as in (21); see Figures 1(a1), $1(\mathrm{a} 2)$, and $1(\mathrm{a} 3)$.

Proposition 5. The global mode point of the negative-ordered Lognormal random variable $X_{\gamma} \sim \mathscr{L} \mathscr{N}_{\gamma}(\mu, \sigma), \gamma \in(-\infty, 0)$, is (in limit) the threshold 0 which is an infinite (probability) density point. Moreover, the location parameter (i.e., $e^{\mu}$ ) of $\mathscr{L} \mathscr{N}_{\gamma}(\mu, \sigma)$ is a nonsmooth (local) mode point for all $X_{\gamma<0}$ that corresponds to locally maximum density

$$
f_{X_{\gamma}}\left(e^{\mu}\right)=\frac{1}{2 \sigma((\gamma-1) / \gamma)^{1 / \gamma} \Gamma((\gamma-1) / \gamma) e^{\mu}},
$$

while $e^{\mu-\sigma^{\gamma}}$ is a local minimum (probability) density point with corresponding locally minimum density $f_{X_{\gamma}}\left(e^{\mu-\sigma^{\gamma}}\right)$.

Proof. The negative-ordered Lognormals are formed by density functions admitting threshold 0 (in limit) for their global mode point (of infinite density), as shown in (12). Moreover, from the previously discussed monotonicity of $f_{X_{\gamma}}$ in Proposition 4, all the negative-ordered Lognormals admit also $e^{\mu}$ as a local nonsmooth mode point and $\exp \left\{\mu-\sigma^{\gamma}\right\}$ as a local minimum density point, with densities as in (26) and $f_{X_{\gamma}}\left(e^{\mu-\sigma^{\gamma}}\right)$, respectively; see Figures $1(\mathrm{~b} 1), 1(\mathrm{~b} 2)$, and 1(b3).

Furthermore, Proposition 5 holds (in limit) for random variables $X_{1}$ and $X_{ \pm \infty}$ which provide Log-Uniform and LogLaplace distributions, respectively. Indeed, for given $a, b \in$ $\mathbb{R}_{+}^{*}, X_{1} \sim \mathscr{L} \mathcal{U}(a, b)=\mathscr{L} \mathcal{N}_{1}(\mu, \sigma)$ with $\mu=(1 / 2) \log (a b)$ and $\sigma=(1 / 2) \log (b / a)$, we get, through (20) and (21), that Mode $X_{1}:=$ Mode $X_{\gamma \rightarrow 1^{+}}=e^{\mu-\sigma}=a$ with corresponding maximum density (i.e., the maximum value of the density function),

$$
\begin{aligned}
\max f_{X_{1}} & =f_{X_{1}}(a)=\frac{e^{\sigma-\mu}}{2 \sigma} \lim _{\gamma \rightarrow 1^{+}} \frac{((\gamma-1) / \gamma)^{(\gamma-1) / \gamma}}{\Gamma(((\gamma-1) / \gamma)+1)} \\
& =\frac{1}{a \log (b / a)} .
\end{aligned}
$$

Moreover, the nonzero minimum density (i.e., the minimum, but not zero, value of the density function) is obtained at $x=b$ with $\min f_{X_{1}}=f_{X_{1}}(b)=1 / 2 \sigma b=1 /(b \log (b / a))$. These results are in accordance with the Log-Uniform density function in (14).

For $X_{ \pm \infty} \sim \mathscr{L} \mathcal{N}_{ \pm \infty}(\log \mu, 1 / \sigma)=\mathscr{L} \mathscr{L}(\mu, \sigma, \sigma)$, we evaluate, through (20) and (21), that

$$
\operatorname{Mode} X_{+\infty}= \begin{cases}0, & \sigma<1, \\ \frac{\mu}{e} & \sigma=1, \\ \mu, & \sigma>1,\end{cases}
$$

with the corresponding maximum density value being infinite; that is, $\max f_{X_{\gamma}}=f_{X_{\gamma}}(0)=+\infty$, provided $\sigma<1$, and 

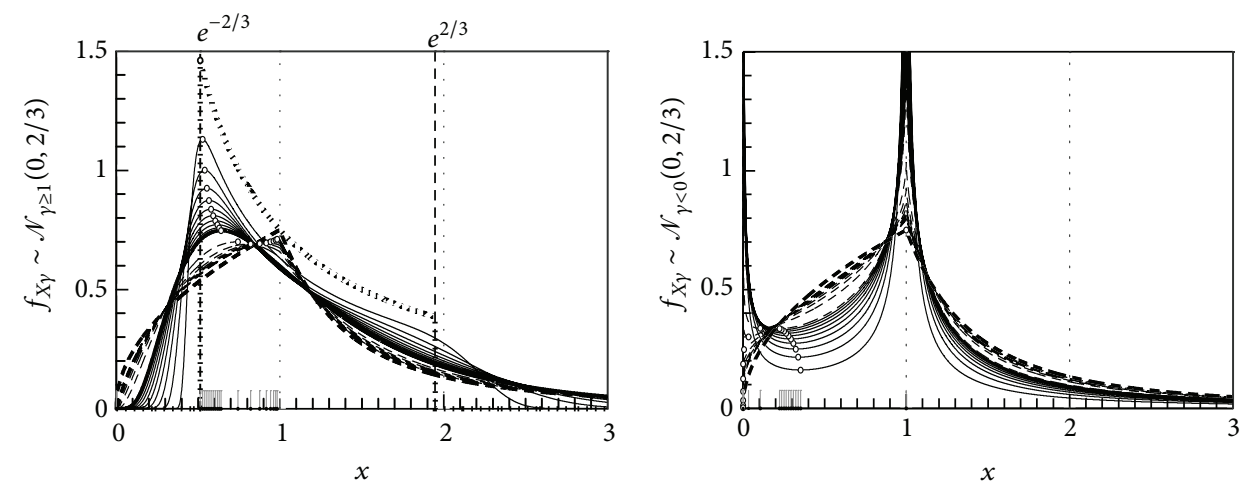

$$
\begin{aligned}
& \ldots X_{1} \sim \mathscr{L} \mathscr{U}\left(e^{-2 / 3}, e^{2 / 3}\right) \\
& -X_{1.1,1.2, \ldots, 1.9} \\
& -X_{2} \sim \mathscr{L} \mathscr{N}(0,2 / 3) \\
& ---X_{3,4, \ldots, 10} \\
& ---X_{+\infty} \sim \mathscr{L} \mathscr{L}(1,3 / 2,3 / 2)
\end{aligned}
$$

$$
\begin{aligned}
& --X_{-\infty} \sim \mathscr{L} \mathscr{L}(1,3 / 2,3 / 2) \\
& ---X_{-10,-9, \ldots,-1} \\
& -X_{-0.9,-0.8, \ldots,-0.1}
\end{aligned}
$$

(a1)

(b1)
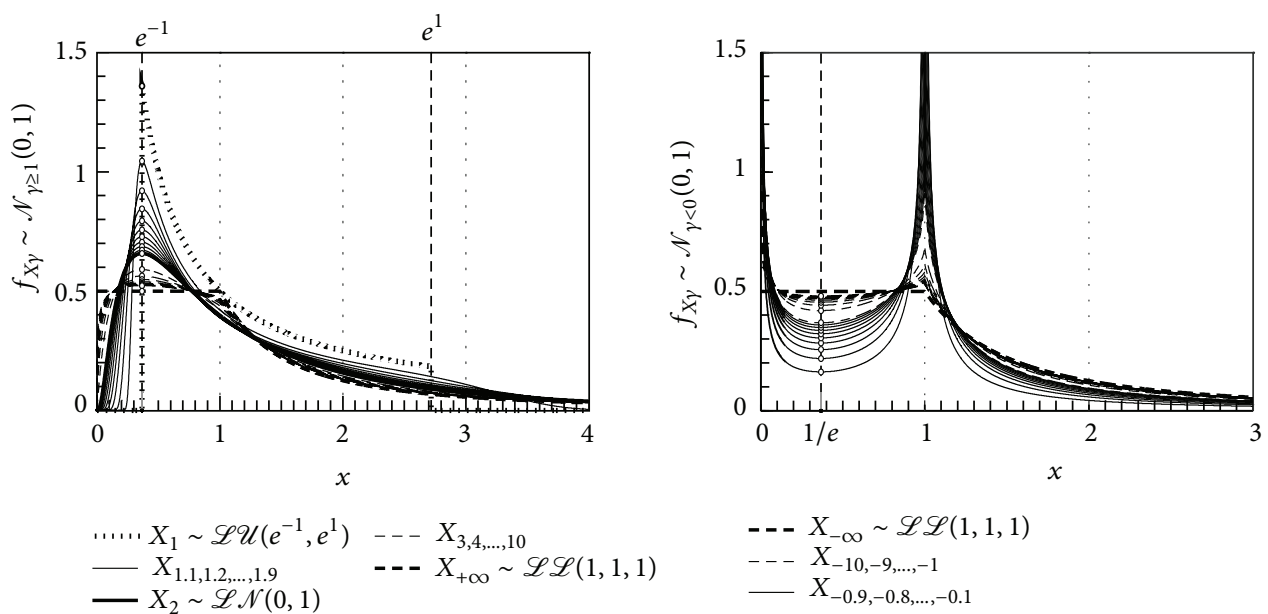

(a2)

(b2)
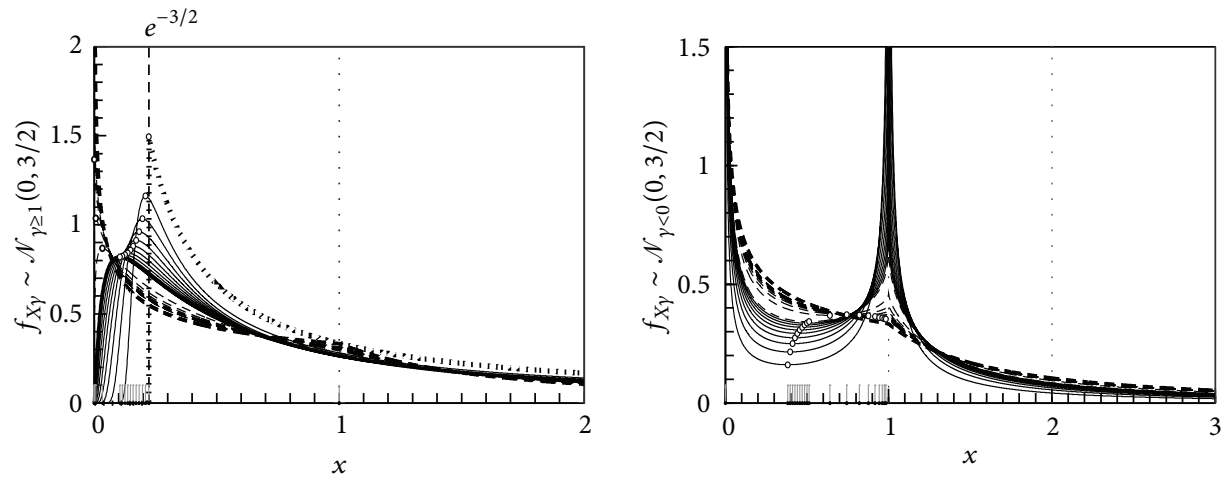

$$
\begin{aligned}
& \ldots X_{1} \sim \mathscr{L} \mathscr{U}\left(e^{-3 / 2}, e^{3 / 2}\right) \\
& -X_{1.1,1.2, \ldots, 1.9} \\
& -X_{2} \sim \mathscr{L} \mathscr{N}(0,3 / 2) \\
& ---X_{3,4, \ldots, 10} \\
& ---X_{+\infty} \sim \mathscr{L} \mathscr{L}(1,2 / 3,2 / 3)
\end{aligned}
$$

(a3)

(b3)

FIGURE 1: Graphs of the density functions $f_{X_{\gamma}}, X_{\gamma} \sim \mathscr{L} \mathscr{N}_{\gamma}(0, \sigma)$, for $\sigma=2 / 3,1,3 / 2$, and various positive (left subfigures) and negative (right subfigures) $\gamma$ values. 
$\max f_{X_{+\infty}}=\sigma /(2 \mu)$, provided $\sigma \geq 1$. The same result can also be derived through (26) as $\gamma \rightarrow-\infty$. These results are in accordance with the Log-Laplace density function in (15), although for $\sigma=1$, Mode $_{ \pm \infty}$ can be defined, through (15), for any value inside the interval $(0, \mu]$.

The above discussion on behavior of the modes with respect to shape parameter $\gamma$ is formed in the following propositions.

Proposition 6. Consider the positive-ordered Lognormal family of distributions $\mathscr{L} \mathcal{N}_{\gamma}(\mu, \sigma)$ with fixed parameters $\mu, \sigma$ and $\gamma \geq 1$. When $\gamma$ rises, that is, when one moves from Log-Uniform to Log-Laplace distribution inside the $\mathscr{L} \mathscr{N}_{\gamma}$ family, the mode points of $\mathscr{L} \mathscr{N}_{\gamma}$ are

(i) strictly increasing from $e^{\mu-\sigma}$ (Log-Uniform case) to $e^{\mu}$ (Log-Laplace case) provided that $\sigma<1$ (with their corresponding maximum density values moving smoothly from $(1 / 2 \sigma) e^{\sigma-\mu}$ to $\left.+\infty\right)$,

(ii) fixed at $e^{\mu-1}$ for all $\mathscr{L} \mathcal{N}_{\gamma \geq 1}(\mu, \sigma=1)$ (with the corresponding maximum density values moving smoothly from $(1 / 2) e^{1-\mu}$ to $\left.(1 / 2) e^{-\mu}\right)$,

(iii) strictly decreasing from $e^{\mu-\sigma}$ (Log-Uniform case) to threshold 0 (Log-Laplace case) provided that $\sigma>1$ (with their corresponding maximum density values moving smoothly from $(1 / 2 \sigma) e^{\sigma-\mu}$ to $\left.(1 / 2 \sigma) e^{-\mu}\right)$.

Proof. Let $X_{\gamma} \sim \mathscr{L} \mathcal{N}_{\gamma}(\mu, \sigma)$, Mode $X_{\gamma}$ is a smooth monotonous function of $\gamma \in(-\infty, 0) \cup(1,+\infty)$ for positive-and negative-ordered $X_{\gamma}$, as

$$
\frac{d}{d \gamma} \operatorname{Mode} X_{\gamma}=-\sigma^{\gamma} \log (\sigma) e^{\mu-\sigma^{\gamma}} .
$$

For $X_{ \pm \infty} \sim \mathscr{L} \mathcal{N}_{ \pm \infty}(\mu, \sigma)$ we evaluate, through (20) and (21), that

$$
\text { Mode } X_{+\infty}= \begin{cases}e^{\mu}, & \sigma<1, \\ e^{\mu-1}, & \sigma=1, \\ 0, & \sigma>1,\end{cases}
$$

with the corresponding maximum density value being infinite; that is, $\max f_{X_{\gamma}}=f_{X_{\gamma}}(0)=+\infty$, provided $\sigma>1$, and $\max f_{X_{+\infty}}=1 /\left(2 \sigma e^{\mu}\right)$, provided $\sigma \leq 1$.

Assume that $\gamma \geq 1$. Considering (27), (30) with (29) and Proposition 4, the results for the positive-ordered Lognormals hold.

Proposition 7. For the negative-ordered Lognormal family of distributions $\mathscr{L} \mathscr{N}_{\gamma}(\mu, \sigma)$ with $\gamma<0$, when $\gamma$ rises, that is, when one moves from Log-Laplace to degenerate Dirac distribution inside the $\mathscr{L} \mathcal{N}_{\gamma}$ family, the local minimum (probability) density points of $\mathscr{L} \mathscr{N}_{\gamma}$ are

(i) strictly increasing from threshold 0 (Log-Laplace case) to $e^{\mu-1}$ (Dirac case) provided that $\sigma<1$,

(ii) fixed at $e^{\mu-1}$ for all $\mathscr{L} \mathcal{N}_{\gamma}(\mu, \sigma=1)$,

(iii) strictly decreasing from $e^{\mu}$ (Log-Laplace case) to $e^{\mu-1}$ (Dirac case) provided that $\sigma>1$.
Proof. Assume now that $\gamma<0$. From (29) we have $(d / d \gamma)$ $\left(e^{\mu-\sigma^{\gamma}}\right)<0$ when $\sigma>1$ and $(d / d \gamma)\left(e^{\mu-\sigma^{\gamma}}\right)>0$ when $\sigma<1$. Therefore, the local minimum density point $e^{\mu-\sigma^{\gamma}}$ (see Proposition 5) for $\sigma>1$ is decreasing from $\left.e^{\mu-\sigma^{\gamma}}\right|_{\gamma \rightarrow-\infty}=e^{\mu}$ to Mode $X_{0}=e^{\mu-1}$ through (20). When $\sigma=1, e^{\mu-\sigma^{\gamma}}=$ $e^{\mu-1}$ for all $\gamma<0$, while for $\sigma<1, e^{\mu-\sigma^{\gamma}}$ increases from $\left.e^{\mu-\sigma^{\gamma}}\right|_{\gamma \rightarrow-\infty}=0$ to Mode $X_{0}=e^{\mu-1}$ through (20).

It is easy to see that for the $\log$-Laplace case $\mathscr{L} \mathscr{L}(\mu, \sigma, \sigma)$, the local minimum density point $e^{\mu-\sigma^{\gamma}}$ of $X_{\gamma<0}$ with $\sigma>1$ coincides (in limit) with the local nonsmooth mode point $e^{\mu}$ of $X_{\gamma}$; see Figure 1(b3). Also, notice that the local minimum density point $e^{\mu-\sigma^{\gamma}}, \gamma<0$, for the Dirac case $\mathscr{D}\left(e^{\mu}\right)$, is the limiting point $e^{\mu-1}$ although the (probability) density in $\mathscr{D}\left(e^{\mu}\right)$ case vanishes everywhere except at the infinite pole $e^{\mu}$.

Figure 1 illustrates the probability density functions $f_{X_{\gamma}}$ curves for scale parameters $\sigma=2 / 3,1,3 / 2$ of the positiveordered lognormally distributed $X_{\gamma} \sim \mathscr{L} \mathscr{N}_{\gamma \geq 1}(0, \sigma)$ in Figures 1(a1)-1(a3), respectively, while the p.d.f. of negativeordered lognormally distributed $X_{\gamma} \sim \mathscr{L} \mathcal{N}_{\gamma<0}(0, \sigma)$ are depicted in Figures 1(b1)-1(b3), respectively. Moreover, the density points $e^{\mu-\sigma^{\gamma}}$ on $f_{X_{\gamma}}$ are also depicted (small circles over p.d.f. curves with their corresponding ticks on $x$-axis). According to Proposition 7, in Figures 1(a1)-1(a3), that is, for positive-ordered $X_{\gamma \geq 1}$, these density points represent the mode points on $f_{X_{\gamma}}$ while in Figures 1(b1)-1(b3), that is, for negative-ordered $X_{\gamma<0}$, represent the local minimum density points on $f_{X_{\gamma}}$ curves.

For the evaluation of the cumulative distribution function (c.d.f.) of the generalized Lognormal distribution, the following theorem is stated and proved.

Theorem 8. The c.d.f. $F_{X_{\gamma}}$ of a $\gamma$-order Lognormal random variable $X_{\gamma} \sim \mathscr{L} \mathscr{N}_{\gamma}(\mu, \sigma)$ is given by

$$
\begin{aligned}
& F_{X_{\gamma}}(x) \\
& =\frac{1}{2}+\frac{\sqrt{\pi}}{2 \Gamma((\gamma-1) / \gamma) \Gamma(\gamma /(\gamma-1))} \\
& \times \operatorname{Erf}_{\gamma /(\gamma-1)}\left\{\left(\frac{\gamma-1}{\gamma}\right)^{(\gamma-1) / \gamma} \frac{\log x-\mu}{\sigma}\right\} \\
& =1-\frac{1}{2 \Gamma((\gamma-1) / \gamma)} \Gamma\left(\frac{\gamma-1}{\gamma}, \frac{\gamma-1}{\gamma}\left(\frac{\log x-\mu}{\sigma}\right)^{\gamma /(\gamma-1)}\right) \text {, } \\
& x \in \mathbb{R}_{+}^{*} .
\end{aligned}
$$

Proof. From density function $f_{X \gamma}$, as in (10), we have

$$
\begin{aligned}
F_{X_{\gamma}}(x) & =F_{X_{\gamma}}(x ; \mu, \sigma, \gamma)=\int_{0}^{x} f_{X_{\gamma}}(t) d t \\
& =\sigma^{-1} C_{\gamma}^{1} \int_{0}^{x} t^{-1} \exp \left\{-\frac{\gamma-1}{\gamma}\left|\frac{\log t-\mu}{\sigma}\right|^{\gamma /(\gamma-1)}\right\} d t .
\end{aligned}
$$


Applying the transformation $w=(\log t-\mu) / \sigma, t>0$, the above c.d.f. is reduced to

$$
\begin{aligned}
F_{X_{\gamma}}(x) & =C_{\gamma}^{1} \int_{-\infty}^{(\log x-\mu) / \sigma} \exp \left\{-\frac{\gamma-1}{\gamma}|w|^{\gamma /(\gamma-1)}\right\} d w \\
& =\Phi_{Z_{\gamma}}\left(\frac{\log x-\mu}{\sigma}\right),
\end{aligned}
$$

where $\Phi_{Z_{\gamma}}$ is the c.d.f. of the standardized $\gamma$-order normal distribution $Z_{\gamma}=(1 / \sigma)\left(\log X_{\gamma}-\mu\right) \sim \mathcal{N}_{\gamma}(0,1)$. Moreover, $\Phi_{Z_{\gamma}}$ can be expressed in terms of the generalized error function. In particular,

$$
\begin{aligned}
\Phi_{Z_{\gamma}}(z) & =C_{\gamma}^{1} \int_{-\infty}^{z} \exp \left\{-\frac{\gamma-1}{\gamma}|w|^{\gamma /(\gamma-1)}\right\} d w \\
& =\Phi_{Z_{\gamma}}(0)+C_{\gamma}^{1} \int_{0}^{z} \exp \left\{-\frac{\gamma-1}{\gamma}|w|^{\gamma /(\gamma-1)}\right\} d w,
\end{aligned}
$$

and as $f_{Z_{\gamma}}$ is a symmetric density function around zero, we have

$$
\begin{aligned}
\Phi_{Z_{\gamma}}(z) & =\frac{1}{2}+C_{\gamma}^{1} \int_{0}^{z} \exp \left\{-\frac{\gamma-1}{\gamma}|w|^{\gamma /(\gamma-1)}\right\} d w \\
& =\frac{1}{2}+C_{\gamma}^{1} \int_{0}^{z} \exp \left\{-\left|\left(\frac{\gamma-1}{\gamma}\right)^{(\gamma-1) / \gamma} w\right|^{\gamma /(\gamma-1)}\right\} d w,
\end{aligned}
$$

and thus

$$
\begin{aligned}
\Phi_{Z_{\gamma}}(z)= & \frac{1}{2}+C_{\gamma}^{1}\left(\frac{\gamma}{\gamma-1}\right)^{(\gamma-1) / \gamma} \\
& \times \int_{0}^{((\gamma-1) / \gamma)^{(\gamma-1) / \gamma} z} \exp \left\{-u^{\gamma /(\gamma-1)}\right\} d u .
\end{aligned}
$$

Substituting the normalizing factor, as in (2), and using (3), we obtain

$$
\begin{aligned}
\Phi_{Z_{\gamma}}(z)= & \frac{1}{2}+\frac{\sqrt{\pi}}{2 \Gamma(((\gamma-1) / \gamma)+1) \Gamma((2 \gamma-1) /(\gamma-1))} \\
& \times \operatorname{Erf}_{\gamma /(\gamma-1)}\left\{\left(\frac{\gamma-1}{\gamma}\right)^{(\gamma-1) / \gamma} z\right\}, \quad z \in \mathbb{R},
\end{aligned}
$$

and finally, through (34), we derive (31), which forms (32) through (4).

It is essential for numeric calculations to express (31) considering positive arguments for Erf. Indeed, through (37), we have

$$
\begin{aligned}
F_{X_{\gamma}}(x)= & \frac{1}{2}+\frac{\operatorname{sgn}(\log x-\mu) \sqrt{\pi}}{2 \Gamma((\gamma-1) / \gamma) \Gamma(\gamma /(\gamma-1))} \\
& \times \operatorname{Erf}_{\gamma /(\gamma-1)}\left\{\left(\frac{\gamma-1}{\gamma}\right)^{(\gamma-1) / \gamma}\left|\frac{\log x-\mu}{\sigma}\right|\right\},
\end{aligned}
$$

while applying (4) into (39) it is obtained that

$$
\begin{aligned}
F_{X_{\gamma}}(x)= & \frac{1+\operatorname{sgn}(\log x-\mu)}{2}-\frac{\operatorname{sgn}(\log x-\mu)}{2 \Gamma((\gamma-1) / \gamma)} \\
& \times \Gamma\left(\frac{\gamma-1}{\gamma}, \frac{\gamma-1}{\gamma}\left|\frac{\log x-\mu}{\sigma}\right|^{\gamma /(\gamma-1)}\right) .
\end{aligned}
$$

As the generalized error function $\operatorname{Erf}_{a}$ is defined in (4), through the upper incomplete gamma function $\Gamma\left(a^{-1}, \cdot\right)$, series expansions can be used for a more "numericaloriented" form of (4). Here some expansions of the c.d.f. of the generalized Lognormal distribution are presented.

Corollary 9. The c.d.f. $F_{X_{\gamma}}$ can be expressed in the series expansion form

$$
\begin{array}{r}
F_{X_{\gamma}}(x)=\frac{1}{2}+\frac{((\gamma-1) / \gamma)^{(\gamma-1) / \gamma}}{(2 / \gamma) \Gamma((\gamma-1) / \gamma)}\left(\frac{\log x-\mu}{\sigma}\right) \\
\times \sum_{k=0}^{\infty} \frac{\left(((1-\gamma) / \gamma)|(\log x-\mu) / \sigma|^{\gamma /(\gamma-1)}\right)^{k}}{k ![(k+1) \gamma-1]}, \\
x \in \mathbb{R}_{+}^{*} .
\end{array}
$$

Proof. Substituting the series expansion form of (6) into (39) and expressing the infinite series using the integer powers $k$, the series expansion as in (41) is derived.

Corollary 10. For the negative-ordered lognormally distributed random variable $X_{\gamma}$ with $\gamma=1 /(1-n) \in \mathbb{R}_{-}, n \in \mathbb{N}$, $n \geq 2$, the finite expansion is obtained as

$$
\begin{aligned}
F_{X_{\nu}}(x)= & \frac{1}{2}+\frac{1}{2} \operatorname{sgn}(\log x-\mu)-\frac{\operatorname{sgn}(\log x-\mu)}{2 \exp \left\{n|(\log x-\mu) / \sigma|^{1 / n}\right\}} \\
& \times \sum_{k=0}^{n-1} \frac{n^{k}}{k !}\left|\frac{\log x-\mu}{\sigma}\right|^{k / n} .
\end{aligned}
$$

Proof. Applying the following finite expansion form of the upper incomplete gamma function,

$$
\Gamma(n, x)=(n-1) ! e^{-x} \sum_{k=0}^{n-1} \frac{x^{k}}{k}, \quad x \in \mathbb{R}, n \in \mathbb{N}^{*}=\mathbb{N} \backslash 0,
$$

into (40), we readily get (42).

Example 11. For the (-1)-ordered lognormally distributed $X_{-1}$ (i.e., for $n=2$ ), we have

$$
\begin{aligned}
F_{X_{-1}}(x)= & \frac{1}{2}+\frac{1}{2} \operatorname{sgn}(\log x-\mu)-\operatorname{sgn}(\log x-\mu) \\
& \times \frac{1+2 \sqrt{|(\log x-\mu) / \sigma|}}{2 \exp \{2 \sqrt{|(\log x-\mu) / \sigma|}\}},
\end{aligned}
$$


while for the $(-1 / 2)$-ordered lognormally distributed $X_{-1 / 2}$ (i.e., for $n=3$ ), we have

$$
\begin{aligned}
F_{X_{-1 / 2}}(x)= & \frac{1}{2}+\frac{1}{2} \operatorname{sgn}(\log x-\mu)-\operatorname{sgn}(\log x-\mu) \\
& \times \frac{1+3 \sqrt[3]{|(\log x-\mu) / \sigma|}+9 \sqrt[3]{((\log x-\mu) / \sigma)^{2}}}{2 \exp \{3 \sqrt[3]{|(\log x-\mu) / \sigma|}\}} .
\end{aligned}
$$

Example 12. For the second-ordered Lognormal random variable $X_{2} \sim \mathscr{L} \mathscr{N}_{2}(\mu, \sigma)$, we immediately derive, from (31), that

$$
\begin{aligned}
F_{X_{2}}(x) & =\Phi_{X_{2}}\left(\frac{\log x-\mu}{\sigma}\right)=\frac{1}{2}+\frac{1}{2} \operatorname{Erf}_{2}\left(\frac{\log x-\mu}{\sqrt{2} \sigma}\right) \\
& =\frac{1}{2}+\frac{1}{2} \operatorname{erf}\left(\frac{\log x-\mu}{\sqrt{2} \sigma}\right) ;
\end{aligned}
$$

that is, the c.d.f. of the usual Lognormal is derived, as it is expected, due to $\mathscr{L} \mathcal{N}_{2}=\mathscr{L} \mathcal{N}$; see Theorem 3 .

Example 13. For the infinite-ordered Lognormal $X_{ \pm \infty} \sim$ $\mathscr{L} \mathcal{N}_{ \pm \infty}(\mu, \sigma)$, setting $(\gamma-1) / \gamma=1$, we obtain through (41) and the exponential series expansion that

$$
\begin{aligned}
F_{X_{ \pm \infty}} & (x) \\
= & \frac{1}{2}+\frac{1}{2} \sqrt{\pi} \operatorname{Erf}_{1}\left(\frac{\log x-\mu}{\sigma}\right) \\
= & \frac{1}{2}-\frac{1}{2} \operatorname{sgn}(\log x-\mu) \sum_{k=0}^{\infty} \frac{1}{(k+1) !}\left(-\left|\frac{\log x-\mu}{\sigma}\right|\right)^{k+1} \\
= & \frac{1}{2}+\frac{1}{2} \operatorname{sgn}(\log x-\mu)-\frac{1}{2} \operatorname{sgn}(\log x-\mu) \\
& \times \exp \left\{-\left|\frac{\log x-\mu}{\sigma}\right|\right\},
\end{aligned}
$$

and hence

$$
F_{X_{ \pm \infty}}(x)= \begin{cases}\frac{1}{2} e^{\mu / \sigma} x^{1 / \sigma}, & x \in\left(0, e^{\mu}\right], \\ 1-\frac{e^{\mu / \sigma}}{2 x^{1 / \sigma}}, & x \in\left(e^{\mu},+\infty\right),\end{cases}
$$

which is the c.d.f. of the Log-Laplace distribution as in (15). This is expected as $\mathscr{L} \mathscr{N}_{ \pm \infty}(\mu, \sigma)=\mathscr{L} \mathscr{L}\left(e^{\mu}, 1 / \sigma, 1 / \sigma\right)$; see Theorem 3.

It is interesting to mention here that the same result can also be derived through (42), as this finite expansion can be extended for $n=1$, which provides (in limit) the c.d.f. of the infinite-ordered Lognormal distribution.
Example 14. Similarly, for the first-ordered random variable $X_{1} \sim \mathscr{L} \mathcal{N}_{1}(\mu, \sigma)$, the expansion (41) can be written as

$$
\begin{aligned}
& F_{X_{\gamma}}(x) \\
& =\frac{1}{2}+\frac{((\gamma-1) / \gamma)^{(\gamma-1) / \gamma}}{2 \Gamma(((\gamma-1) / \gamma)+1)}\left(\frac{\log x-\mu}{\sigma}\right) \\
& \quad \times\left[1+(\gamma-1) \sum_{k=1}^{\infty} \frac{\left(((1-\gamma) / \gamma)|(\log x-\mu) / \sigma|^{\gamma /(\gamma-1)}\right)^{k}}{k ![(k+1) \gamma-1]}\right],
\end{aligned}
$$

and provided that $(\log x-\mu) / \sigma \leq 1$, we obtain

$$
\begin{aligned}
F_{X_{1}}(x) & =\lim _{\gamma \rightarrow 1^{+}} F_{X_{\gamma}}(x)=\frac{1}{2}+\frac{\log x-\mu}{2 \sigma}(1+0) \\
& =\frac{\log x-\mu+\sigma}{2 \sigma},
\end{aligned}
$$

with $F_{X_{1}}\left(e^{\mu-\sigma}\right)=0$ and $F_{X_{1}}\left(e^{\mu+\sigma}\right)=1$. Therefore,

$$
F_{X_{1}}(x)= \begin{cases}0, & x \in\left(0, e^{\mu-\sigma}\right), \\ \frac{1}{2 \sigma}(\log x-\mu+\sigma), & x \in\left[e^{\mu-\sigma}, e^{\mu+\sigma}\right], \\ 1, & x \in\left(e^{\mu+\sigma},+\infty\right),\end{cases}
$$

coincides with the c.d.f. of the Log-Uniform distribution $\mathscr{L} \mathcal{U}\left(a=e^{\mu-\sigma}, b=e^{\mu+\sigma}\right)$ as in (15). This is expected as $\mathscr{L} \mathcal{N}_{1}(\mu, \sigma)=\mathscr{L} \mathscr{U}\left(e^{\mu-\sigma}, e^{\mu+\sigma}\right)$; see Theorem 3 .

Table 1 provides the probability values $P_{\gamma ; 1}=\operatorname{Pr}\left\{X_{\gamma} \leq i\right\}$, $i=1 / 2,1,2, \ldots, 5$, for various $X_{\gamma} \sim \mathscr{L N}_{\gamma}(0,1)$. Notice that $P_{\gamma ; 1}=1 / 2$ for all $\gamma$ values due to the fact that $1=\left.e^{\mu}\right|_{\mu=0}=$ Med $X_{\gamma}$ (see Theorem 8); that is, the point 1 coincides with the $\gamma$-invariant median of the $\mathscr{L} \mathcal{N}_{\gamma}(0,1)$ family discussed previously. Moreover, the last two columns provide also the 1st and 3rd quartile points $q_{1 ; \gamma}$ and $q_{3 ; \gamma}$ of $X_{\gamma}$; that is, $\operatorname{Pr}\left\{X_{\gamma} \leq\right.$ $\left.q_{k ; \gamma}\right\}=k / 4, k=1,3$, for various $\gamma$ values. These quartiles are evaluated using the quantile function $\mathrm{Q}_{X_{\nu}}$ of r.v. $X_{\gamma}$; that is,

$$
\begin{aligned}
& \mathrm{Q}_{X_{\gamma}}(P):=\inf \left\{x \in \mathbb{R}_{+}^{*} \mid F_{X_{\gamma}}(x) \geq P\right\} \\
& =\exp \{\operatorname{sgn}(2 P-1) \sigma \\
& \left.\times\left[\frac{\gamma}{\gamma-1} \Gamma^{-1}\left(\frac{\gamma-1}{\gamma},|2 P-1|\right)\right]^{(\gamma-1) / \gamma}\right\},
\end{aligned}
$$$$
P \in(0,1) \text {, }
$$

for $P=1 / 4,3 / 4$, that is derived through (40). The values of the inverse upper incomplete gamma function $\Gamma^{-1}((\gamma-1) / \gamma, \cdot)$ were numerically calculated.

Figure 2 illustrates the c.d.f. $F_{X_{\gamma}}$ curves, as in (39), for certain r.v. $X_{\gamma} \sim \mathscr{L} \mathcal{N}_{\gamma}(0, \sigma)$ and for scale parameters $\sigma=2 / 3$, 
TABLE 1: Probability mass values $P_{\gamma ; i}=\operatorname{Pr}\left\{X_{\gamma} \leq i\right\}, i=1 / 2,1,2, \ldots, 5$, and the 1 st and 3 rd quartiles $q_{1 ; \gamma}, q_{3 ; \gamma}$ for various generalized lognormally distributed $X_{\gamma} \sim \mathscr{L} \mathscr{N}_{\gamma}(0,1)$.

\begin{tabular}{lcccccccc}
\hline$\gamma$ & $P_{\gamma ; 1 / 2}$ & $P_{\gamma ; 1}$ & $P_{\gamma ; 2}$ & $P_{\gamma ; 3}$ & $P_{\gamma ; 4}$ & $P_{\gamma ; 5}$ & $q_{1 ; \gamma}$ & $q_{3 ; \gamma}$ \\
\hline-50 & 0.2501 & 0.5000 & 0.7499 & 0.8326 & 0.8739 & 0.8987 & 0.4998 & 2.0008 \\
-10 & 0.2505 & 0.5000 & 0.7495 & 0.8297 & 0.8698 & 0.8940 & 0.4990 & 2.0038 \\
-5 & 0.2508 & 0.5000 & 0.7492 & 0.8264 & 0.8652 & 0.8887 & 0.4982 & 2.0071 \\
-2 & 0.2515 & 0.5000 & 0.7485 & 0.8187 & 0.8539 & 0.8756 & 0.4964 & 2.0145 \\
-1 & 0.2521 & 0.5000 & 0.7479 & 0.8097 & 0.8408 & 0.8601 & 0.4945 & 2.0223 \\
$-1 / 2$ & 0.2524 & 0.5000 & 0.7476 & 0.7989 & 0.8248 & 0.8410 & 0.4925 \\
$-1 / 10$ & 0.2528 & 0.5000 & 0.7482 & 0.7757 & 0.7895 & 0.7984 & 0.4986 \\
1 & 0.1534 & 0.5000 & 0.8466 & 1.0000 & 1.0000 & 1.0000 & 0.6065 \\
$3 / 2$ & 0.2381 & 0.5000 & 0.7619 & 0.8848 & 0.9437 & 0.9721 & 0.5172 \\
2 & 0.2441 & 0.5000 & 0.7559 & 0.8640 & 0.9172 & 0.9462 & 0.5092 \\
3 & 0.2472 & 0.5000 & 0.7528 & 0.8505 & 0.8989 & 0.9267 & 0.5049 \\
4 & 0.2481 & 0.5000 & 0.7519 & 0.8452 & 0.8917 & 0.9188 & 0.5034 \\
5 & 0.2486 & 0.5000 & 0.7514 & 0.8425 & 0.8878 & 0.9145 & 0.5025 \\
10 & 0.2494 & 0.5000 & 0.7506 & 0.8375 & 0.8810 & 0.9068 & 1.9630 \\
50 & 0.2499 & 0.5000 & 0.7501 & 0.8341 & 0.8761 & 0.9013 & 1.9867 \\
$\pm \infty$ & 0.2500 & 0.5000 & 0.7500 & 0.8333 & 0.8750 & 0.9000 & 0.5011 & 1.9954 \\
\hline
\end{tabular}

$1,3 / 2$ in the 3 subfigures, respectively. Moreover, the 1st and 3rd quartile points $\mathrm{Q}_{X_{\gamma}}(1 / 4)$ and $\mathrm{Q}_{X_{\gamma}}(3 / 4)$ are also depicted (small circles over c.d.f. curves with their corresponding ticks on $x$-axis).

Theorem 15. The (non-log-scaled) location parameter $e^{\mu}$ is in fact the geometric mean as well as the median for all generalized lognormally distributed $X_{\gamma} \sim \mathscr{L}_{\mathcal{N}}(\mu, \sigma)$. Moreover, this median is also characterized by vanishing median absolute deviation.

Proof. Considering (39) and the fact that $\operatorname{Erf}_{a} 0=0, a \in \mathbb{R}_{+}^{*}$, it holds that $\operatorname{Med} X_{\gamma}=F_{X_{\gamma}}^{-1}(1 / 2)=e^{\mu}$. For the geometric mean $\left(\mu_{g}\right)_{X_{\gamma}}=e^{\mathrm{E}\left[\log X_{\gamma}\right]}$, we readily obtain $\left(\mu_{g}\right)_{X_{\gamma}}=e^{\mu}$ as $\log X_{\gamma} \sim \mathcal{N}_{\gamma}\left(\mu, \sigma^{2}\right)$ with $\mathrm{E}\left[X_{\gamma}\right]=\mu$. A dispersion measure for the median is the so-called median absolute deviation or $\operatorname{MAD}$, defined by $\operatorname{MAD}\left(X_{\gamma}\right)=\operatorname{Med}\left|X_{\gamma}-\operatorname{Med} X_{\gamma}\right|$. For $X_{\gamma} \sim \mathscr{L} \mathcal{N}_{\gamma}(\mu, \sigma)$, we have $X_{\gamma}-\operatorname{Med} X_{\gamma}=X_{\gamma}-e^{\mu} \sim$ $\mathscr{L} \mathscr{N}_{\gamma}\left(\mu, \sigma ;-e^{\mu}\right)$; that is, $X_{\gamma}-e^{\mu}$ follows the generalized Lognormal distribution with threshold $-e^{\mu}$. Furthermore, $|Y|$ is the "folded distribution" case of $Y:=X_{\gamma}-e^{\mu}$ which is distributed through p.d.f. of the form

$$
f_{|Y|}(x)=f_{Y}(-x)+f_{Y}(x), \quad x \in \mathbb{R}_{+}
$$

where $f_{Y}$ is the p.d.f. of $Y$. For example, see [17] on the folded normal distribution. However, the density function $f_{Y}$ is defined in $\left(-e^{\mu},+\infty\right)$ due to threshold $-e^{\mu}$, while it vanishes elsewhere; that is,

$$
f_{|Y|}(x)= \begin{cases}f_{Y}(-x)+f_{Y}(x), & 0 \leq x \leq e^{\mu}, \\ f_{Y}(x), & x>e^{\mu} .\end{cases}
$$

Therefore, the c.d.f. of $|Y|$ is given by

$$
\begin{aligned}
F_{|Y|}(x)=\int_{0}^{x} f_{|Y|}(t) d t= & \int_{0}^{e^{\mu}} f_{Y}(-t) d t+\int_{0}^{e^{\mu}} f_{Y}(t) d t \\
& +\int_{e^{\mu}}^{x} f_{Y}(t) d t, \quad x \in \mathbb{R}_{+} .
\end{aligned}
$$

Applying the transformation $w=(1 / \sigma)\left[\log \left(e^{\mu}-t\right)-\mu\right], t<$ $e^{\mu}$, into the first integral of (55) and $z=(1 / \sigma)\left[\log \left(t+e^{\mu}\right)-\mu\right]$, $t>-e^{\mu}$, into the other two integrals, we obtain

$$
\begin{gathered}
F_{|Y|}(x)=C_{\gamma}^{1} \int_{-\infty}^{0} \exp \left\{-\frac{\gamma-1}{\gamma}|w|^{\gamma /(\gamma-1)}\right\} d w+C_{\gamma}^{1} \\
\quad \times \int_{0}^{(1 / \sigma) \log 2} \exp \left\{-\frac{\gamma-1}{\gamma}|z|^{\gamma /(\gamma-1)}\right\} d z \\
+C_{\gamma}^{1} \int_{(1 / \sigma) \log 2}^{g(x)} \exp \left\{-\frac{\gamma-1}{\gamma}|z|^{\gamma /(\gamma-1)}\right\} d z, \\
g(x):=\frac{\log \left(x+e^{\mu}\right)-\mu}{\sigma}, \quad x \in \mathbb{R}_{+},
\end{gathered}
$$

and hence

$$
\begin{aligned}
F_{|Y|}(x)= & \Phi_{Z}(0)+\left[\Phi_{Z}\left(\frac{\log 2}{\sigma}\right)-\Phi_{Z}(0)\right] \\
& +\left[\Phi_{Z}(g(x))-\Phi_{Z}\left(\frac{\log 2}{\sigma}\right)\right] \\
= & \Phi_{Z}\left(\frac{\log \left(x+e^{\mu}\right)-\mu}{\sigma}\right),
\end{aligned}
$$




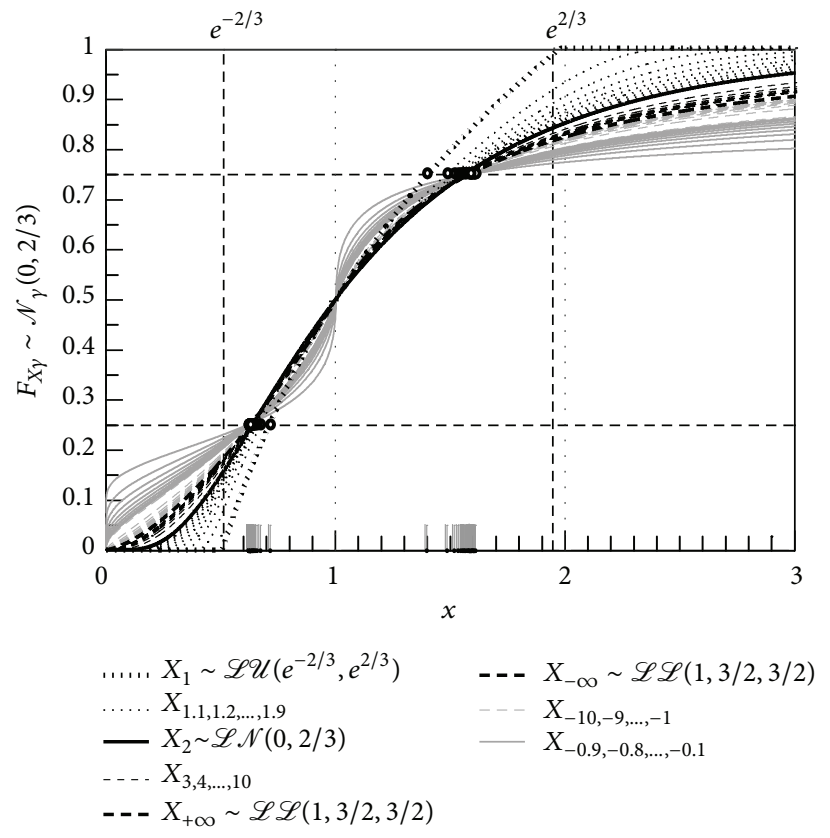

(a)

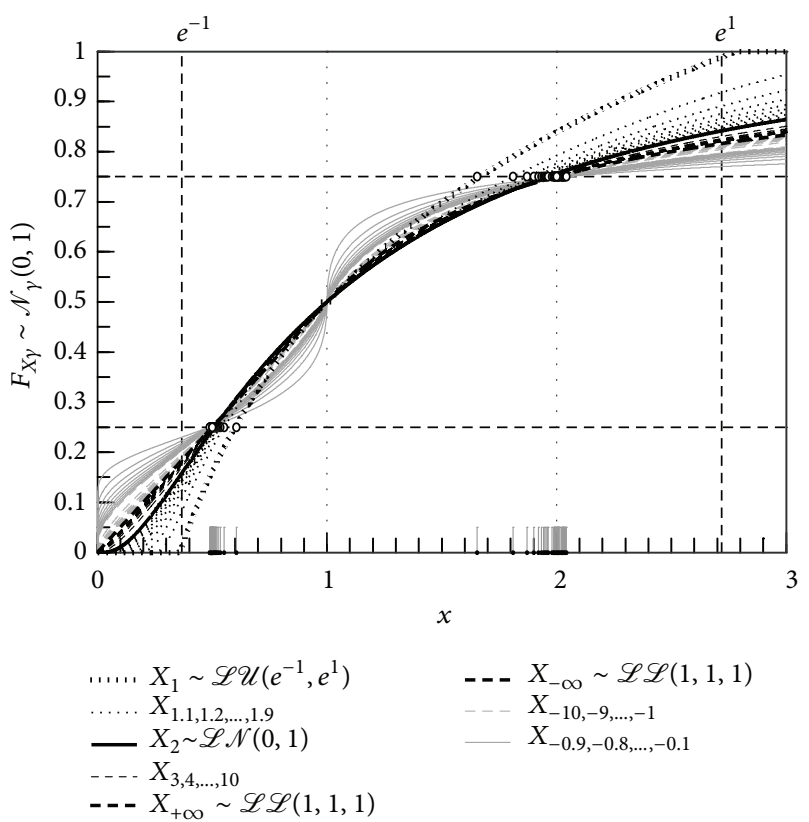

(b)

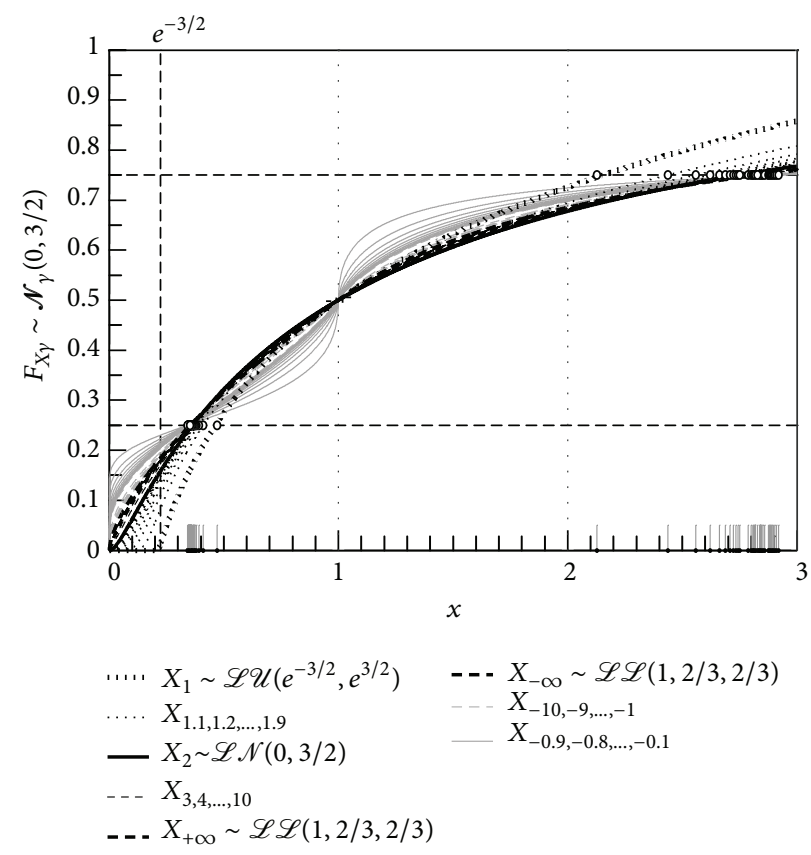

(c)

FIGURE 2: Graphs of the c.d.f. $F_{X_{\gamma}}, X_{\gamma} \sim \mathscr{L} \mathscr{N}_{\gamma}(0, \sigma)$, for $\sigma=2 / 3,1,3 / 2$, and various $\gamma$ values.

with $\Phi_{Z}$ being the c.d.f. of the standardized r.v. $Z \sim \mathcal{N}_{\gamma}(0,1)$. From (38) and the fact that $\operatorname{Erf}_{a} 0=0, a \in \mathbb{R}_{+}^{*}$, it is clear that (57) implies $\operatorname{MADX}_{\gamma}=\operatorname{Med}\left|X_{\gamma}-e^{\mu}\right|=F_{\left|X_{\gamma}-e^{\mu}\right|}^{-1}(1 / 2)=0$, for every $X_{\gamma} \sim \mathscr{L} \mathcal{N}_{\gamma}(\mu, \sigma)$, and the theorem has been proved.

\section{Moments of the $\gamma$-Order Lognormal Distribution}

For the evaluation of the moments of the generalized Lognormal distribution, the following holds. 
Proposition 16. The th raw moment $\tilde{\mu}_{X}^{(t)}$ of a generalized lognormally distributed random variable $X \sim \mathscr{L} \mathscr{N}_{\gamma}(\mu, \sigma)$ is given by

$$
\begin{aligned}
\widetilde{\mu}_{X}^{(t)}= & \frac{e^{t \mu}}{\Gamma((\gamma-1) / \gamma)} \sum_{n=0}^{\infty} \frac{(t \sigma)^{2 n}}{(2 n) !}\left(\frac{\gamma}{\gamma-1}\right)^{2 n((\gamma-1) / \gamma)} \\
& \times \Gamma\left((2 n+1) \frac{\gamma-1}{\gamma}\right)
\end{aligned}
$$

and coincides with the moment generating function of the $\gamma$ order normally distributed $\log X$; that is, $M_{\log X}(t)=\widetilde{\mu}_{X}^{(t)}$.

Proof. From the definition of the $t$ th raw moment $\tilde{\mu}_{X}^{(t)}$, we have

$$
\begin{aligned}
\tilde{\mu}_{X}^{(t)} & =\mathrm{E}\left[X^{t}\right]=\int_{\mathbb{R}_{+}} x^{t} f_{X}(x) d x \\
& =\frac{1}{\sigma} C_{\gamma}^{1} \int_{\mathbb{R}_{+}} x^{t-1} \exp \left\{-\frac{\gamma-1}{\gamma}\left|\frac{\log x-\mu}{\sigma}\right|^{\gamma /(\gamma-1)}\right\} d x,
\end{aligned}
$$

and applying the transformation $z=((\gamma-1) / \gamma)^{(\gamma-1) / \gamma}(1 / \sigma)$ $(\log x-\mu), x>0$, we get

$$
\begin{aligned}
\tilde{\mu}_{X}^{(t)}=C_{\gamma}^{1}\left(\frac{\gamma}{\gamma-1}\right)^{(\gamma-1) / \gamma} \int_{\mathbb{R}} & \exp \left\{t \mu+n\left(\frac{\gamma}{\gamma-1}\right)^{(\gamma-1) / \gamma} \sigma z\right\} \\
& \times \exp \left\{-|z|^{\gamma /(\gamma-1)}\right\} d z .
\end{aligned}
$$

Through the exponential series expansion

$$
\exp \left\{t\left(\frac{\gamma}{\gamma-1}\right)^{(\gamma-1) / \gamma} \sigma z\right\}=\sum_{n=0}^{\infty} \frac{(t \sigma)^{n}}{n !}\left(\frac{\gamma}{\gamma-1}\right)^{n((\gamma-1) / \gamma)} z^{n},
$$

it is obtained that

$$
\begin{aligned}
\widetilde{\mu}_{X}^{(t)}= & 2 C_{\gamma}^{1}\left(\frac{\gamma}{\gamma-1}\right)^{(\gamma-1) / \gamma} e^{t \mu} \sum_{n=0}^{\infty} \frac{(t \sigma)^{2 n}}{(2 n) !}\left(\frac{\gamma}{\gamma-1}\right)^{2 n((\gamma-1) / \gamma)} \\
& \times \int_{\mathbb{R}_{+}} z^{2 n} \exp \left\{-z^{\gamma /(\gamma-1)}\right\} d z .
\end{aligned}
$$

Finally, substituting the normalizing factor $C_{\gamma}^{1}$ as in (2) into (62) and utilizing the known integral [16],

$$
\int_{\mathbb{R}+} x^{m} e^{-b x^{n}} d x=\frac{\Gamma((m+1) / n)}{n b^{(m+1) / n}}, \quad n, m, b \in \mathbb{R}_{+}^{*},
$$

we obtain (58).

Moreover, for $Y:=\log X \sim \mathcal{N}_{\gamma}\left(\mu, \sigma^{2}\right)$ we have $M_{Y}(t)=$ $\mathrm{E}\left[e^{t Y}\right]=\mathrm{E}\left[X^{t}\right]=\widetilde{\mu}_{X}^{(t)}$, and the proposition has been proved.
Example 17. For the second-ordered lognormally distributed $X \sim \mathscr{L} \mathcal{N}_{2}(\mu, \sigma),(58)$ implies

$$
\widetilde{\mu}_{X}^{(t)}=\frac{e^{t \mu}}{\sqrt{\pi}} \sum_{n=0}^{\infty} \frac{\left(2 t^{2} \sigma^{2}\right)^{n}}{(2 n) !} \Gamma\left(n+\frac{1}{2}\right), \quad t \in \mathbb{R}_{+},
$$

and through the gamma function identity

$$
\Gamma\left(n+\frac{1}{2}\right)=\frac{(2 n) !}{2^{2 n} n !} \sqrt{\pi}, \quad n \in \mathbb{N},
$$

we have

$$
\begin{aligned}
\tilde{\mu}_{X}^{(t)} & =e^{t \mu} \sum_{n=0}^{\infty} \frac{(t \sigma)^{2 n}}{2^{n} n !}=e^{t \mu} \sum_{n=0}^{\infty} \frac{1}{n !}\left(\frac{1}{2} t^{2} \sigma^{2}\right)^{n} \\
& =e^{t \mu+(1 / 2)(t \sigma)^{2}}, \quad t \in \mathbb{R}_{+},
\end{aligned}
$$

which is the $t$ th raw moment of the usual lognormally distributed $X \sim \mathscr{L} \mathscr{N}(\mu, \sigma)$, with mean $\mu_{X}:=\widetilde{\mu}_{X}^{(1)}=\mathrm{E}[X]=$ $\exp \left\{\mu+(1 / 2) \sigma^{2}\right\}$. This is true as $M_{\log X}(t)=\widetilde{\mu}_{X}^{(t)}=\exp \{t \mu+$ $\left.(1 / 2)(t \sigma)^{2}\right\}$ is the known moment-generating function of the normally distributed $\log X \sim \mathcal{N}\left(\mu, \sigma^{2}\right)$.

Theorem 18. The kth central moment (about the mean) $\mu_{X}^{(t)}$ of a generalized lognormally distributed random variable $X \sim$ $\mathscr{L} \mathscr{N}_{\gamma}(\mu, \sigma)$ is given by

$$
\mu_{X}^{(k)}=\frac{e^{k \mu}}{\Gamma((\gamma-1) / \gamma)} \sum_{n=0}^{k}\left(\begin{array}{l}
k \\
n
\end{array}\right)\left(-\frac{\mu_{X}}{e^{\mu}}\right)^{n} S_{k-n}, \quad k \in \mathbb{N},
$$

where

$$
\begin{array}{r}
S_{k}=\sum_{m=0}^{\infty} \frac{(k \sigma)^{2 m}}{(2 m) !}\left(\frac{\gamma}{\gamma-1}\right)^{2 m((\gamma-1) / \gamma)} \Gamma\left((2 m+1) \frac{\gamma-1}{\gamma}\right), \\
k \in \mathbb{N} .
\end{array}
$$

Proof. From the definition of the $k$ th central moment $\mu_{X}^{(k)}$ we have

$$
\mu_{X}^{(k)}:=\mathrm{E}\left[\left(X-\mu_{X}\right)^{k}\right]=\int_{\mathbb{R}_{+}}\left(x-\mu_{X}\right)^{k} f_{X}(x ; \mu, \sigma, \gamma) d x,
$$

while using the binomial identity we get

$$
\begin{aligned}
\mu_{X}^{(k)} & =\sum_{n=0}^{k}\left(\begin{array}{l}
k \\
n
\end{array}\right)\left(-\mu_{X}\right)^{n} \int_{\mathbb{R}_{+}} x^{k-n} f_{X}(x) d x \\
& =\sum_{n=0}^{k}\left(\begin{array}{l}
k \\
n
\end{array}\right)\left(-\mu_{X}\right)^{n} \widetilde{\mu}_{X}^{(k-n)} .
\end{aligned}
$$

Applying Proposition 16, (70) implies that

$$
\begin{aligned}
\mu_{X}^{(k)}= & \frac{e^{k \mu}}{\Gamma((\gamma-1) / \gamma)} \sum_{n=0}^{k}\left(\begin{array}{l}
k \\
n
\end{array}\right)\left(-\frac{\mu_{X}}{e^{\mu}}\right)^{n} \sum_{m=0}^{\infty} \frac{[(k-n) \sigma]^{2 m}}{(2 m) !} \\
& \times\left(\frac{\gamma}{\gamma-1}\right)^{2 m((\gamma-1) / \gamma)} \Gamma((2 m+1)((\gamma-1) / \gamma)),
\end{aligned}
$$


while taking the summation index $n$ until $k-1$, we finally obtain (67), and the theorem has been proved.

Example 19. Recall Example 17. Substituting (66) and the mean $\mu_{X}=e^{\mu+(1 / 2) \sigma^{2}}$ into (70), the second-ordered lognormally distributed $X \sim \mathscr{L} \mathscr{N}_{2}(\mu, \sigma)$ provides

$$
\mu_{X}^{(k)}=\sum_{n=0}^{k}\left(\begin{array}{l}
k \\
n
\end{array}\right)(-1)^{n} e^{k \mu+(1 / 2)\left[n+(k-n)^{2}\right] \sigma^{2}}, \quad k \in \mathbb{N},
$$

while

$$
\sigma_{X}^{2}:=\operatorname{Var}[X]=\mu_{X}^{(2)}=e^{2 \mu+\sigma^{2}}\left(e^{\sigma^{2}}-1\right)
$$

which are the $k$ th central moment and the variance, respectively, of the usual lognormally distributed $X \sim \mathscr{L} \mathcal{N}(\mu, \sigma)$. The same result can be derived directly through (67) for $\gamma=2$ and the use of the known gamma function identity, as in (65).

Theorem 20. The mean $\mu_{X}:=\mathrm{E}[X]$, variance $\sigma_{X}^{2}:=\operatorname{Var}[X]$, coefficient of variation $C V_{X}$, skewness $\lambda_{X}$ and kurtosis $\kappa_{X}$ of the generalized lognormally distributed $X \sim \mathscr{L} \mathscr{N}_{\gamma}(\mu, \sigma)$ are, respectively, given by

$$
\begin{aligned}
\mu_{X} & =\frac{e^{\mu}}{\Gamma((\gamma-1) / \gamma)} S_{1} \\
\sigma_{X}^{2} & =-\mu_{X}^{2}+\frac{e^{2 \mu}}{\Gamma((\gamma-1) / \gamma)} S_{2} \\
C V_{X}^{2} & =\Gamma\left(\frac{\gamma-1}{\gamma}\right) \frac{S_{2}}{S_{1}^{2}}-1 \\
\lambda_{X} & =-C V_{X}^{-3}-C V_{X}^{-1}+\frac{e^{3 \mu}}{\sigma_{X}^{3} \Gamma((\gamma-1) / \gamma)} S_{3} \\
\kappa_{X} & =-C V_{X}^{-4}-6 C V_{X}^{-2}-4 \frac{\lambda_{X}}{C V_{X}}+\frac{e^{4 \mu}}{\sigma_{X}^{4} \Gamma((\gamma-1) / \gamma)} S_{4},
\end{aligned}
$$

where the sums $S_{i}, i=1, \ldots, 4$, are given by (68).

Proof. From Proposition 16 we easily obtain (74), as $\mu_{X}:=$ $\tilde{\mu}_{X}^{(1)}$. From Theorem 18 we have

$$
\sigma_{X}^{2}:=\mu_{X}^{(2)}=\mu_{X}^{2}+\left[\Gamma\left(\frac{\gamma-1}{\gamma}\right)\right]^{-1}\left(e^{2 \mu} S_{2}-2 e^{\mu} \mu_{X} S_{1}\right) .
$$

Hence, substituting $S_{1}$ from (74), (75) holds. Moreover, the squared coefficient of variation is readily obtained via (75) and (74). By definition, skewness $\lambda_{X}$ is the standardized third (central) moment; that is, $\lambda_{X}:=\operatorname{Skew}[X]=\mu_{X}^{(3)} / \sigma_{X}^{3}$. Theorem 18 provides that

$$
\begin{aligned}
\lambda_{X}= & -C V_{X}^{-3}+\left[\sigma_{X}^{3} \Gamma\left(\frac{\gamma-1}{\gamma}\right)\right]^{-1} \\
& \times\left(e^{3 \mu} S_{3}-3 e^{2 \mu} \mu_{X} S_{2}+3 e^{\mu} \mu_{X} S_{1}\right) .
\end{aligned}
$$

Substituting $S_{1}$ and $S_{2}$ from (74) and (75), we obtain (77). Finally, kurtosis $\kappa_{X}$ is (by definition) the standardized fourth (central) moment; that is, $\kappa_{X}:=\operatorname{Kurt}[X]=\mu_{X}^{(4)} / \sigma_{X}^{4}$, which provides, through Theorem 18 , that

$$
\begin{aligned}
\kappa_{X}= & C V_{X}^{-4}+\left[\sigma_{X}^{4} \Gamma\left(\frac{\gamma-1}{\gamma}\right)\right]^{-1} \\
& \times\left(e^{4 \mu} S_{4}-4 e^{3 \mu} \mu_{X} S_{3}+6 e^{2 \mu} \mu_{X}^{2} S_{2}-4 e^{\mu} \mu_{X}^{3} S_{1}\right) .
\end{aligned}
$$

Substituting $S_{i}, i=1,2,3$, from (74), (75), and (77), we obtain (78).

Example 21. For the second-ordered lognormally distributed $X \sim \mathscr{L} \mathscr{N}_{2}(\mu, \sigma)$, utilizing (65) into (68) we get $S_{n}=$ $\sqrt{\pi} e^{\left(n^{2} \sigma^{2}\right) / 2}, n \in \mathbb{N}^{*}$. Applying this to Theorem 20 we derive (after some algebra)

$$
\begin{gathered}
\mu_{X}=e^{\mu+(1 / 2) \sigma^{2}}, \quad \sigma_{X}^{2}=e^{2 \mu+\sigma^{2}}\left(e^{\sigma^{2}}-1\right), \\
C V_{X}=\sqrt{e^{\sigma^{2}}-1}, \\
\lambda_{X}=\left(e^{\sigma^{2}}+2\right) \sqrt{e^{\sigma^{2}}-1}, \quad \kappa_{X}=e^{4 \sigma^{2}}+2 e^{3 \sigma^{2}}+3 e^{2 \sigma^{2}}-3,
\end{gathered}
$$

which are the mean, variance, coefficient of variation, skewness, and kurtosis, respectively, of usual lognormally distributed $X \sim \mathscr{L} \mathscr{N}(\mu, \sigma)$.

For the usual lognormally distributed random variable $X \sim \mathscr{L} \mathcal{N}$, it is known that Mode $X<\operatorname{Med} X<\mu_{X}$. The following corollary examines this inequality for the $\mathscr{L} \mathscr{N}_{\gamma}$ family of distributions.

Corollary 22. For the $\gamma$-ordered lognormally distributed $X_{\gamma} \sim$ $\mathscr{L} \mathscr{N}_{\gamma}(\mu, \sigma)$, it is true that Mode $X_{\gamma} \leq \operatorname{Med} X_{\gamma}=\left(\mu_{g}\right)_{X_{\gamma}} \leq$ $\mu_{X_{\gamma}}$. The first equality holds for the Log-Laplace distributed $X_{+\infty}$ with $\sigma<1$ as well as for all the negative-ordered $X_{\gamma<0}$ where Mode $X_{\gamma}$ is considered to be the local (nonsmooth) mode point of $X_{\gamma}$. The second equality holds for the degenerate Dirac case of $X_{0}$.

Proof. From (74) and Theorem 15 we have

$$
\operatorname{Med} X_{\gamma}=\left(\mu_{g}\right)_{X_{\gamma}}=e^{\mu}<\mu_{X_{\gamma}},
$$

for every $X_{\gamma} \sim \mathscr{L} \mathcal{N}_{\gamma}(\mu, \sigma)$. The above inequality becomes equality for the limiting Dirac case of $X_{0}$. For the relation between the mode and the median of $X_{\gamma}$, the following cases are considered.

(i) The positive-ordered Lognormal case $\gamma>1$ : from (20) we have

$$
\text { Mode } X_{\gamma}=e^{\mu-\sigma^{\gamma}}<e^{\mu}=\operatorname{Med} X_{\gamma} .
$$

For the Log-Laplace case of $X_{+\infty}$, it holds

$$
\text { Mode } X_{\gamma}=e^{\mu}=\operatorname{Med} X_{\gamma} \text {, }
$$


provided that $\sigma<1$, while for $\sigma \geq 1$ we have

$$
\text { Mode } X_{\gamma}=0<e^{\mu}=\operatorname{Med} X_{\gamma} \text {. }
$$

For $\sigma=1$, the inequality (84) clearly holds.

(ii) The negative-ordered Lognormal case $\gamma<0$ : from Proposition 4 the inequality as in (86) holds. Moreover, if Mode $X_{\gamma}$ is considered as the nonsmooth local mode point of the negative-ordered $X_{\gamma}$ then the equality as in (85) holds.

From the above cases and (83), the corollary holds true.

Corollary 23. The raw and central moments of a Log-Uniformly distributed random variable $X \sim \mathscr{L} \mathcal{U}(a, b), 0<a<b$, are given by

$$
\begin{aligned}
& \tilde{\mu}_{X}^{(t)}=\frac{b^{t}-a^{t}}{t \log (b / a)}, \quad t \in \mathbb{R}, \\
& \mu_{X}^{(k)}=\frac{(a-b)^{k}}{\log ^{k}(b / a)}+\frac{1}{\log (b / a)} \\
& \times \sum_{n=0}^{k-1}\left(\begin{array}{l}
k \\
n
\end{array}\right) \frac{(a-b)^{n}\left(b^{k-n}-a^{k-n}\right)}{(k-n) \log ^{n}(b / a)}, \quad k \in \mathbb{N},
\end{aligned}
$$

respectively, while the mean, variance, coefficient of variation, skewness, and kurtosis of $X$ are given, respectively, by

$$
\begin{gathered}
\mu_{X}=\frac{b-a}{\log (b / a)}, \\
\sigma_{X}^{2}=\frac{(b-a)^{2}}{\log ^{2}(b / a)}+\frac{(b-a)(b+a)}{2 \log (b / a)}, \\
\lambda_{X}=\frac{1}{\sigma_{X}^{3}}\left[\frac{b^{3}-a^{3}}{3 \log (b / a)}-3 \frac{(b-a)^{2}(b+a)}{2 \log ^{2}(b / a)}+2 \frac{(b-a)^{3}}{\log ^{3}(b / a)}\right], \\
\kappa_{X}=\frac{1+\frac{b+a}{2(b-a)}}{\sigma_{X}^{4}}\left[\frac{b^{4}-a^{4}}{4 \log (b / a)}-4 \frac{(b-a)\left(b^{3}-a^{3}\right)}{3 \log ^{2}(b / a)}\right. \\
\left.+3 \frac{(b-a)^{3}(b+a)}{\log ^{3}(b / a)}-3 \frac{(b-a)^{4}}{\log ^{4}(b / a)}\right] .
\end{gathered}
$$

Proof. Recall Proposition 16 with $X_{\gamma} \sim \mathscr{L} \mathscr{N}_{\gamma}(\mu, \sigma)$. Through the gamma function additive identity (58) can be written as

$$
\begin{aligned}
\tilde{\mu}_{X_{\gamma}}^{(t)}=\frac{e^{t \mu}}{t \sigma \Gamma((\gamma-1) / \gamma+1)} \sum_{m=0}^{\infty} & \frac{(t \sigma)^{2 m+1}}{(2 m+1) !}\left(\frac{\gamma}{\gamma-1}\right)^{2 m((\gamma-1) / \gamma)} \\
& \times \Gamma\left((2 m+1) \frac{\gamma-1}{\gamma}+1\right) .
\end{aligned}
$$

Thus, letting $X:=X_{1} \sim \mathscr{L} \mathcal{N}_{1}(\mu, \sigma)=\mathscr{L} \mathscr{U}(a, b)$ with $\mu=(1 / 2) \log (a b)$ and $\sigma=(1 / 2) \log (b / a)$, it holds (recall the exponential odd series expansion) that

$$
\begin{array}{r}
\tilde{\mu}_{X}^{(t)}=\lim _{\gamma \rightarrow 1^{+}} \mu_{X_{\gamma}}^{(t)}=\frac{e^{t \mu}}{t \sigma} \sum_{m=0}^{\infty} \frac{(t \sigma)^{2 m+1}}{(2 m+1) !}=\frac{e^{t(\mu+\sigma)}-e^{t(\mu-\sigma)}}{2 t \sigma}, \\
t \in \mathbb{R},
\end{array}
$$

and hence (87) holds. Moreover, $\mu_{X}:=\mu_{X}^{(1)}=\mathrm{E}[X]=(1 / 2 \sigma)$ $\left(e^{\mu+\sigma}-e^{\mu-\sigma}\right)$, and therefore (89) holds.

Working similarly, (67) implies

$$
\begin{array}{r}
\mu_{X}^{(k)}=\lim _{\gamma \rightarrow 1^{+}} \mu_{X_{\gamma}}^{(k)}=e^{k \mu} \sum_{n=0}^{k}\left(\begin{array}{l}
k \\
n
\end{array}\right)\left(-\frac{\mu_{X}}{e^{\mu}}\right)^{n} \sum_{m=0}^{\infty} \frac{[(k-n) \sigma]^{2 m}}{(2 m+1) !}, \\
k \in \mathbb{N} .
\end{array}
$$

Using the exponential odd series expansion, the above expansion becomes

$$
\mu_{X}^{(k)}=\frac{e^{k \mu}}{2 \sigma} \sum_{n=0}^{k}\left(\begin{array}{l}
k \\
n
\end{array}\right)\left(-\frac{\mu_{X}}{e^{\mu}}\right)^{n} \frac{e^{(k-n) \sigma}-e^{-(k-n) \sigma}}{(k-n)}, \quad k \in \mathbb{N},
$$

and, through (89), we obtain (88). Moreover, for $k=2, \sigma_{X}^{2}:=$ $\operatorname{Var}[X]=\mu_{X}^{(2)}-\mu_{X}^{2}$ implies (90), and hence (91) also holds. For $k=3$ and $k=4$, through $\mu_{X}^{(3)}$ and $\mu_{X}^{(4)}$, we obtain (92) and (93), respectively.

Corollary 24. The raw and central moments of a Log-Laplace distributed random variable $X \sim \mathscr{L} \mathscr{L}(\mu, \sigma, \sigma)$ are given by

$$
\begin{gathered}
\tilde{\mu}_{X}^{(t)}=\frac{\mu^{t} \sigma^{2}}{\sigma^{2}-t^{2}}>\mu^{t}, \quad \sigma>t, t \in \mathbb{R}, \\
\mu_{X}^{(k)}=\mu^{k} \sum_{n=0}^{k}\left(\begin{array}{l}
k \\
n
\end{array}\right) \frac{\sigma^{2(n+1)}}{\left(1-\sigma^{2}\right)^{n}\left[\sigma^{2}-(k-n)^{2}\right]}, \quad \sigma>k, k \in \mathbb{N} .
\end{gathered}
$$

The mean, variance, coefficient of variation, skewness, and kurtosis of $X$ are given, respectively, by

$$
\begin{gathered}
\mu_{X}=\frac{\mu \sigma^{2}}{\sigma^{2}-1}>\mu, \quad \sigma>1, \\
\sigma_{X}^{2}=\frac{\mu^{2} \sigma^{2}\left(2 \sigma^{2}+1\right)}{\left(\sigma^{2}-4\right)\left(\sigma^{2}-1\right)^{2}}, \quad \sigma>2, \\
C V_{X}=\frac{1}{\sigma} \sqrt{\frac{2 \sigma^{2}+1}{\sigma^{2}-4}}, \quad \sigma>2, \\
\lambda_{X}=\frac{2\left(15 \sigma^{4}+7 \sigma^{2}+2\right)}{\sigma\left(\sigma^{2}-9\right)} \sqrt{\frac{\sigma^{2}-4}{\left(2 \sigma^{2}+1\right)^{3}}}, \quad \sigma>3,
\end{gathered}
$$




$$
\kappa_{X}=\frac{3\left(8 \sigma^{8}+212 \sigma^{6}+95 \sigma^{4}+33 \sigma^{2}+12\right)\left(\sigma^{2}-4\right)}{\left(\sigma^{2}-16\right)\left(\sigma^{2}-9\right)\left(2 \sigma^{2}+1\right)^{2}},
$$

$$
\sigma>4
$$

Proof. Let $X_{\gamma} \sim \mathscr{L} \mathscr{L}_{\gamma}(\mu, \sigma, \sigma)=\mathscr{L} \mathscr{N}_{\gamma}(\log \mu, 1 / \sigma, 1 / \sigma)$. For $\gamma= \pm \infty$, that is, $\gamma /(\gamma-1)=1$, the raw moments as in (58) provide

$$
\tilde{\mu}_{X}^{(t)}=\tilde{\mu}_{X_{ \pm \infty}}^{(t)}=\mu^{t} \sum_{k=0}^{\infty}\left(\frac{t}{\sigma}\right)^{2 k}, \quad t \in \mathbb{R},
$$

as $X=X_{ \pm \infty}$, while through the even geometric series expansion, it is

$$
\begin{aligned}
\widetilde{\mu}_{X_{ \pm \infty}}^{(t)} & =\frac{1}{2} \mu^{t}\left[\sum_{k=0}^{\infty}\left(\frac{t}{\sigma}\right)^{k}+\sum_{k=0}^{\infty}\left(-\frac{t}{\sigma}\right)^{k}\right] \\
& =\frac{1}{2} \mu^{t}\left(\frac{\sigma}{\sigma-t}+\frac{\sigma}{\sigma+t}\right),
\end{aligned}
$$

provided that $\sigma>t$, and hence (98) holds. Moreover, $\mu_{X}:=$ $\tilde{\mu}_{X}^{(1)}=\mathrm{E}[X]$, and hence (100) holds.

Working similarly, (67) implies

$$
\mu_{X}^{(k)}=\mu^{k} \sigma^{2} \sum_{n=0}^{k}\left(\begin{array}{l}
k \\
n
\end{array}\right) \frac{\left(-\mu_{X} / \mu\right)^{n}}{\sigma^{2}-(k-n)^{2}}, \quad k \in \mathbb{N},
$$

provided $\sigma>k$, and hence, through (100), the central moments (99) are obtained.

Moreover, for $k=2$ and due to $\sigma_{X}^{2}:=\operatorname{Var}[X]=\tilde{\mu}_{X}^{(2)}-\mu_{X}^{2}$, (101) holds true, while for $k=3$ and $k=4$ we derive, through $\mu_{X}^{(3)}$ and $\mu_{X}^{(4)},(103)$ and (104), respectively.

Example 25. For a uniformly distributed r.v. $U \sim \mathcal{U}(a, b)=$ $\mathcal{N}_{1}(\mu, \sigma)$ with $a=\mu-\sigma$ and $b=\mu+\sigma$, it holds that $\mathrm{LU}:=e^{U} \sim \mathscr{L} \mathcal{U}\left(e^{\mu-\sigma}, e^{\mu+\sigma}\right)$ due to Theorem 3, and therefore $\mathrm{LU}$ is a Log-Uniform distributed r.v. as $\mathrm{LU} \sim \mathscr{L} \mathcal{U}\left(e^{a}, e^{b}\right)$. Applying (87), the known moment-generating function of the uniformly distributed $U \sim \mathcal{U}(a, b)$ is derived; that is, $M_{U}(t):=\mathrm{E}\left[e^{t U}\right]=\tilde{\mu}_{\mathrm{LU}}^{(t)}=\left(e^{t b}-e^{t a}\right)(1 / t(b-a))$.

Similarly, for a Laplace distributed r.v. $L \sim \mathscr{L}(\mu, \sigma)=$ $\mathcal{N}_{ \pm \infty}(\mu, \sigma)$, it holds that LL := $e^{L} \sim \mathscr{L} \mathscr{L}\left(e^{\mu}, 1 / \sigma, 1 / \sigma\right)$ due to Theorem 3 , and therefore LL is a Log-Laplace distributed random variable. Applying (98), we derive the known momentgenerating function of the Laplace distributed $L \sim \mathscr{L}(\mu, \sigma)$; that is, $M_{L}(t):=\mathrm{E}\left[e^{t L}\right]=\tilde{\mu}_{\mathrm{LL}}^{(t)}=e^{t \sigma}\left(1-t^{2} \sigma^{2}\right)^{-1}$.

\section{Conclusion}

The family of the $\gamma$-order Lognormal distributions was introduced, which under certain values of $\gamma$ includes the Log-Uniform, Lognormal, and Log-Laplace distributions as well as the degenerate Dirac distribution. The shape of these distributions for positive and negative shape parameters $\gamma$ as well as the cumulative distribution functions, was extensively discussed and evaluated through corresponding tables and figures. Moreover, a thorough study of moments was carried out, in which nonclosed forms as well as approximations were obtained and investigated in various examples. This generalized family of distributions derived through the family of the $\gamma$-order normal distribution is based on a strong theoretical background as the logarithmic Sobolev inequalities provide. Further examinations and calculations can be produced while an application to real data is upcoming.

\section{Acknowledgment}

The authors would like to thank the referee for his valuable comments that helped improve the quality of this paper.

\section{References}

[1] E. L. Crow and K. Shimizu, Lognormal Distributions, Marcel Dekker, New York, NY, USA, 1988.

[2] A. Parravano, N. Sanchez, and E. J. Alfaro, "The Dependence of prestellar core mass distributions on the structure of the parental cloud," The Astrophysical Journal, vol. 754, no. 2, article $150,2012$.

[3] F. Bernardeau and L. Kofman, "Properties of the cosmological density distribution function," Monthly Notices of the Royal Astronomical Society, vol. 443, pp. 479-498, 1995.

[4] P. Blasi, S. Burles, and A. V. Olinto, "Cosmological magnetic field limits in an inhomogeneous Universe," The Astrophysical Journal Letters, vol. 514, no. 2, pp. L79-L82, 1999.

[5] F. S. Kitaura, "Non-Gaussian gravitational clustering field statistics," Monthly Notices of the Royal Astronomical Society, vol. 420, no. 4, pp. 2737-2755, 2012.

[6] G. Yan and F. B. Hanson, "Option pricing for a stochasticvolatility jump-diffusion model with log-uniform jumpamplitudes," in Proceedings of the American Control Conference, 2006.

[7] T. J. Kozubowski and K. Podgórski, "Asymmetric Laplace distributions," The Mathematical Scientist, vol. 25, no. 1, pp. 3746, 2000.

[8] T. J. Kozubowski and K. Podgórski, "Asymmetric Laplace laws and modeling financial data," Mathematical and Computer Modelling, vol. 34, no. 9-11, pp. 1003-1021, 2001.

[9] M. Geraci and M. Bottai, "Quantile regression for longitudinal data using the asymmetric Laplace distribution," Biostatistics, vol. 8, no. 1, pp. 140-154, 2007.

[10] D. B. Madan, "The variance gamma process and option pricing," The European Financial Review, vol. 2, pp. 79-105, 1998.

[11] C. P. Kitsos and N. K. Tavoularis, "Logarithmic Sobolev inequalities for information measures," IEEE Transactions on Information Theory, vol. 55, no. 6, pp. 2554-2561, 2009.

[12] C. P. Kitsos and N. K. Tavoularis, "New entropy type information measures," in Proceedings of the Information Technology Interfaces (ITI '09), Cavtat, Croatia, June 2009.

[13] C. P. Kitsos and T. L. Toulias, "New information measures for the generalized normal distribution," Information, vol. 1, no. 1, pp. 13-27, 2010.

[14] C. P. Kitsos and T. L. Toulias, "Evaluating information measures for the -order Multivariate Gaussian," in Proceedings by IEEE of the 14th Panhellenic Conference on Informatics (PCI'10), pp. 153157, Tripoli, Greece, September 2010.

[15] C. P. Kitsos, T. L. Toulias, and P. C. Trandafir, "On the multivariate $\gamma$-ordered normal distribution," Far East Journal of Theoretical Statistics, vol. 38, no. 1, pp. 49-73, 2012. 
[16] I. S. Gradshteyn and I. M. Ryzhik, Table of Integrals, Series, and Products, Elsevier, 2007.

[17] F. C. Leone, L. S. Nelson, and R. B. Nottingham, "The folded normal distribution,” Technometrics, vol. 3, pp. 543-550, 1961. 


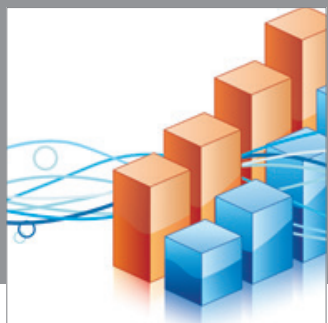

Advances in

Operations Research

mansans

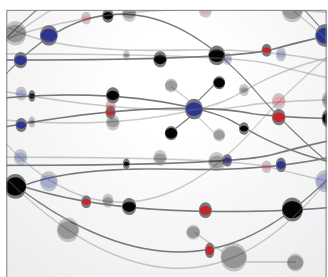

The Scientific World Journal
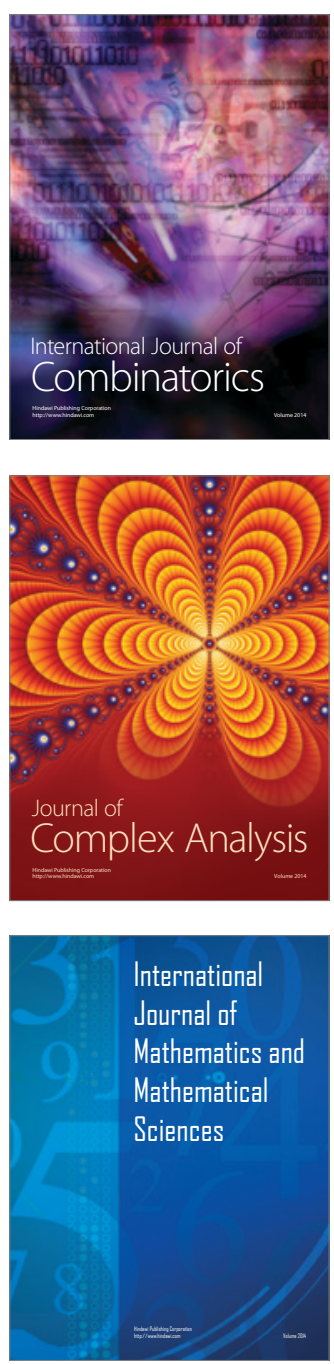
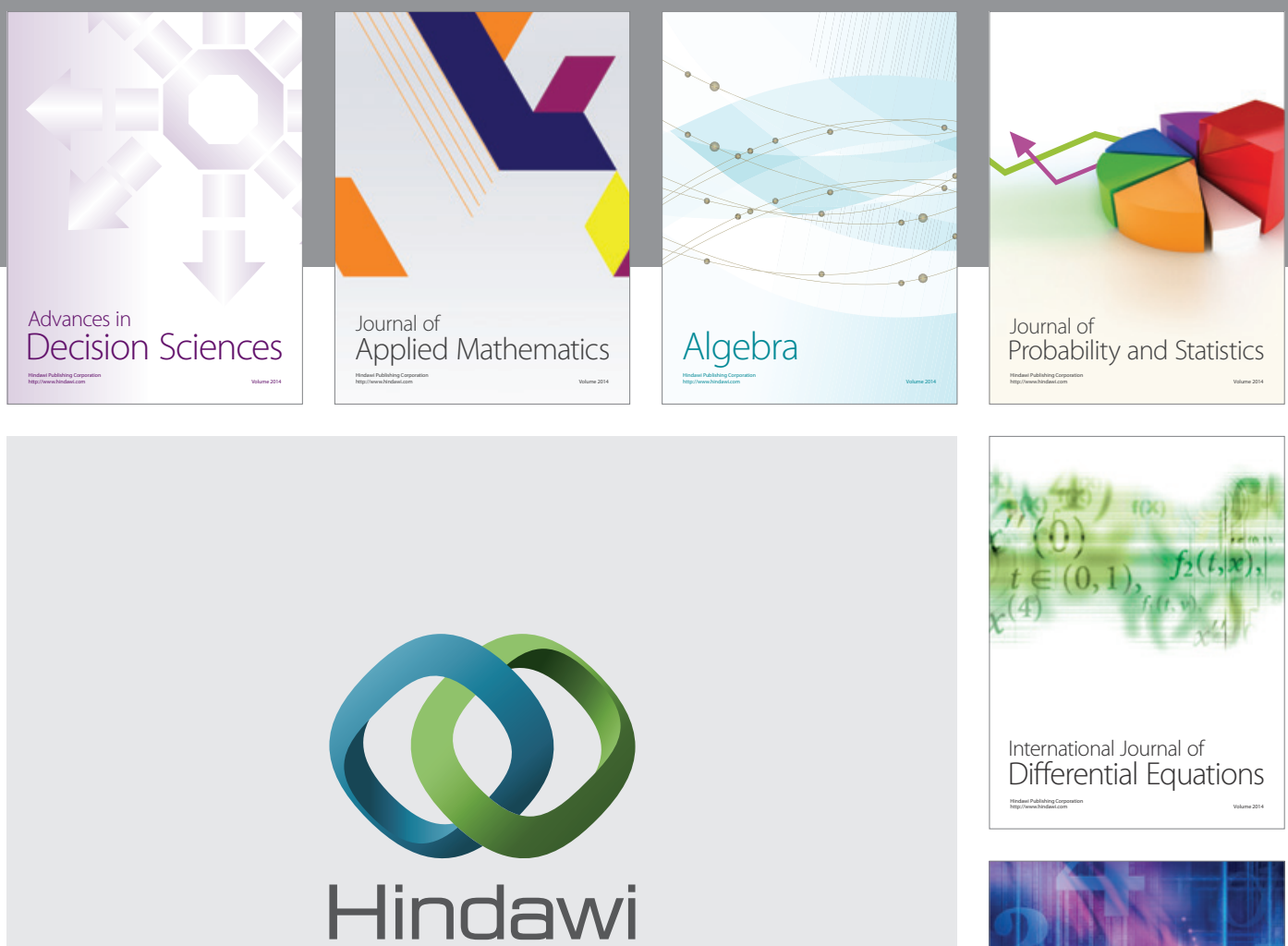

Submit your manuscripts at http://www.hindawi.com
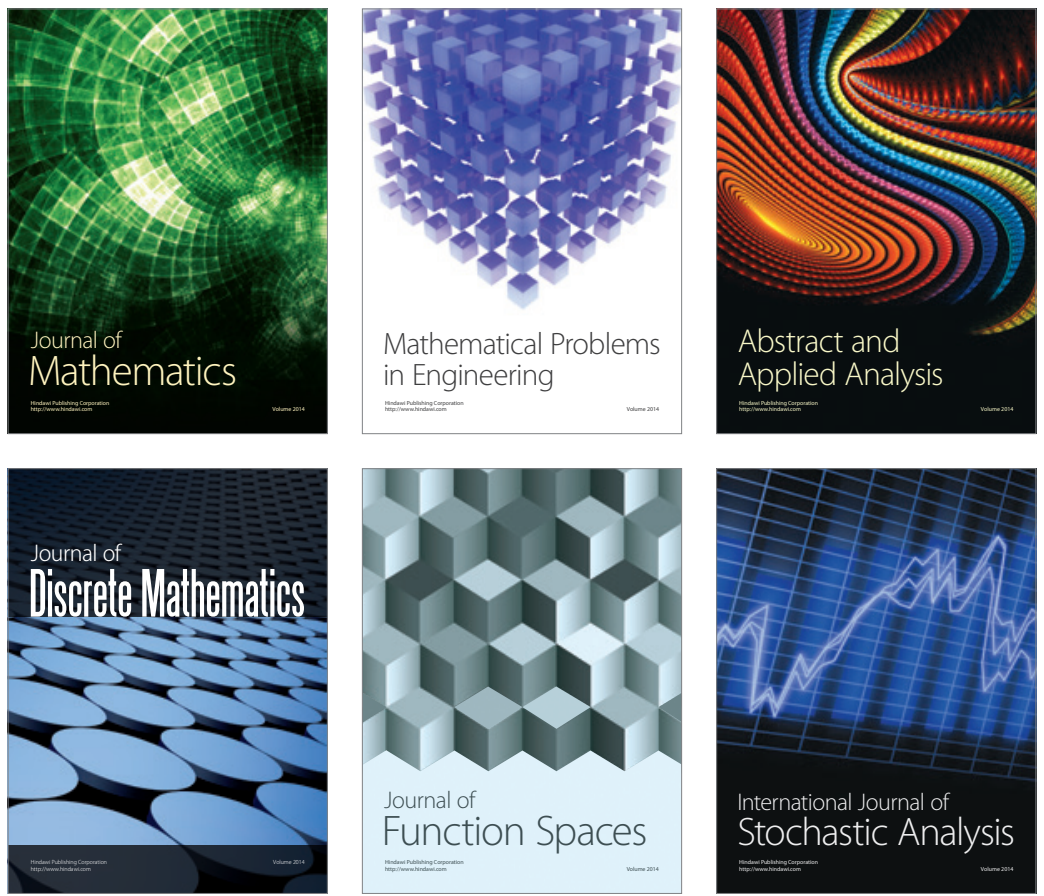

Journal of

Function Spaces

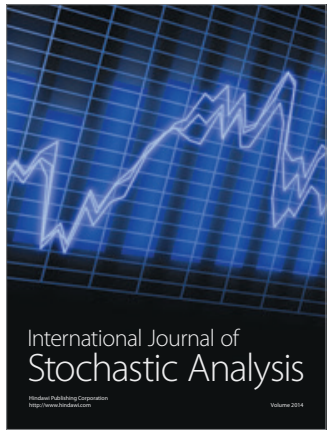

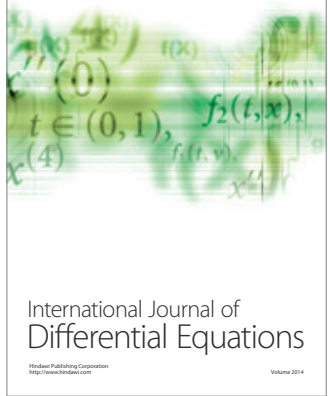
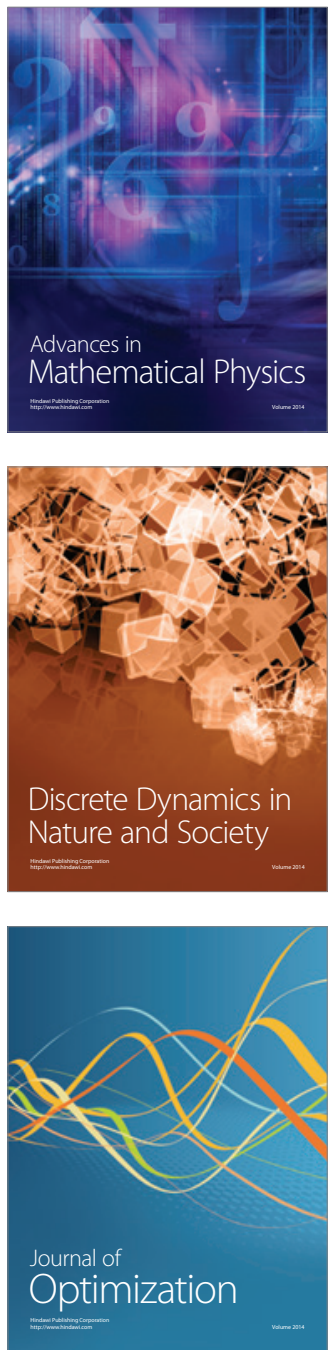